\title{
TITLE:
}

\section{HIGH STEROL ESTER 1 is a key factor in plant sterol homeostasis}

\section{$\operatorname{AUTHOR}(S)$ :}

Shimada, Takashi L.; Shimada, Tomoo; Okazaki, Yozo; Higashi, Yasuhiro; Saito, Kazuki; Kuwata, Keiko; Oyama, Kaori; ... Ueda, Takashi; Takano, Yoshitaka; Hara-Nishimura, Ikuko

\section{CITATION:}

Shimada, Takashi L.... [et al]. HIGH STEROL ESTER 1 is a key factor in plant sterol homeostasis. Nature Plants 2019, 5(11): 1154-1166

\section{ISSUE DATE:}

2019-11

URL:

http://hdl.handle.net/2433/245136

\section{RIGHT:}

This is the accepted manuscript of the article, which has been published in final form at https://doi.org/10.1038/s41477019-0537-2.: The full-text file will be made open to the public on 11 May 2020 in accordance with publisher's 'Terms and Conditions for Self-Archiving'; ; This is not the published version. Please cite only the published version.; この論文は出版 社版でありません。引用の際には出版社版をご確認ご利用ください。 


\section{HIGH STEROL ESTER 1 is a key factor in plant sterol homeostasis}

Takashi L. Shimada1,2,3,4,5, Tomoo Shimada ${ }^{1}$, Yozo Okazaki ${ }^{6,7}$, Yasuhiro Higashi6, Kazuki Saito ${ }^{6,8}$, Keiko Kuwata ${ }^{9}$, Kaori Oyama ${ }^{10}$, Misako Kato ${ }^{10}$, Haruko Ueda ${ }^{11}$, Akihiko Nakano ${ }^{3,12}$, Takashi Ueda ${ }^{4,13,14}$, Yoshitaka Takano², \& Ikuko HaraNishimura ${ }^{1,11^{*}}$

${ }^{1}$ Graduate School of Science, Kyoto University, Sakyo-ku, Kyoto 606-8502, Japan

${ }^{2}$ Graduate School of Agriculture, Kyoto University, Sakyo-ku, Kyoto 606-8502, Japan

${ }^{3}$ Graduate School of Science, The University of Tokyo, Bunkyo-ku, Tokyo 113-0033, Japan

${ }^{4}$ Division of Cellular Dynamics, National Institute for Basic Biology, Okazaki 444-8585, Japan

${ }^{5}$ Graduate School of Horticulture, Chiba University, Matsudo 271-8510, Japan

${ }^{6}$ RIKEN Center for Sustainable Resource Science, Yokohama 230-0045, Japan

${ }^{7}$ Graduate School of Bioresources, Mie University, Tsu 514-8507, Japan

${ }^{8}$ Graduate School of Pharmaceutical Sciences, Chiba University, Chiba 260-8675, Japan

${ }^{9}$ Institute of Transformative Bio-Molecules, Nagoya University, Nagoya 464-8601, Japan

${ }^{10}$ Graduate School of Humanities and Sciences, Ochanomizu University, Tokyo 112-8610, Japan

${ }^{11}$ Faculty of Science and Engineering, Konan University, Kobe 658-8501, Japan

${ }^{12}$ RIKEN Center for Advanced Photonics, Wako 351-0198, Japan

${ }^{13}$ Japan Science and Technology Agency (JST), PRESTO, Kawaguchi 332-0012, Japan

${ }^{14}$ SOKENDAI (Graduate University for Advanced Studies), Okazaki 444-8585, Japan

${ }^{*}$ Correspondence should be addressed to I. H.-N. (e-mail: ihnishi@gr.bot.kyoto-u.ac.jp) 


\section{Abstract}

Plants strictly regulate the sterol levels in their cells, as high sterol levels are toxic. However, how plants achieve sterol homeostasis is not fully understood. We isolated an Arabidopsis thaliana mutant that abundantly accumulated sterol esters in $~ 1-\mu m-$ diameter structures in leaf cells. We designated the mutant as high sterol ester 1 (hise1) and the structures as sterol ester (SE) bodies. Here we show that the gene product (HiSE1) responsible for the mutation functions as a key factor in plant sterol homeostasis on the endoplasmic reticulum (ER) and participates in a fail-safe-regulatory system composed of two processes. First, HiSE1 down-regulates the protein levels of the HMG-CoA reductases HMGR1 and HMGR2, which are rate-limiting enzymes in the sterol synthesis pathway, resulting in suppression of sterol overproduction. Second, if the first process is not successful, excess sterols are converted to sterol esters by phospholipid sterol acyltransferase1 (PSAT1) on the ER microdomains and then segregated in SE bodies.

\section{Introduction}

Sterols have important functions in many cellular activities of plants. For example, brassinosteroids are sterol phytohormones that are involved in cell division, cell elongation, vascular differentiation, and other plant-specific processes. Plant sterols are synthesized from acetyl-CoA via three pathways: the mevalonate, terpenoid, and sterol biosynthetic pathways (see Fig. 3a) ${ }^{1}$. The sterol levels are regulated, often by conversion of excess sterols to sterol esters $^{2,3}$. For example, overexpression of the sterol synthesis enzymes HMG-CoA reductase (HMGR) $^{4}$ and CYP51 $1^{5,6}$ in Nicotiana tabacum leaves induces the accumulation of sterol esters. Application of the sterol precursors squalene and mevalonate to leaves of $A$. thaliana ${ }^{3}$ and Apium graviolens ${ }^{7}$, respectively, also induced the accumulation of sterol-esters.

Deficiency of phospholipid sterol acyltransferase1 (PSAT1), which converts excess sterols into sterol esters ${ }^{2,3}$, causes early senescence of leaves ${ }^{2,3}$, and applying the sterol precursor squalene to PSAT-deficient plants was lethal. In addition, in A. thaliana mutants that accumulate high amounts of cholesterol, the growth of leaves is retarded ${ }^{8,9}$. These results imply that, despite the importance of sterols in plant growth, excess sterols can be harmful. 
Thus, sterol levels should be tightly regulated. However, a key factor regulating the plant sterol homeostasis is largely unknown.

The main sterols of plants, animals and fungi are sitosterol, cholesterol, and ergosterol, respectively ${ }^{10}$. Despite this difference, the metabolic pathway of sterol synthesis of plants is basically similar to those of yeast and animals ${ }^{11}$. HMGR, which is located on endoplasmicreticulum (ER) membrane in eukaryotes ${ }^{11-16}$, is a rate-limiting enzyme that converts HMGCoA to mevalonate in the synthesis of sterols ${ }^{17}$. Thus, its level is an important factor in regulating sterol synthesis. In yeast ${ }^{18}$ and animals ${ }^{19}$, HMGR is degraded through ERassociated degradation (ERAD) ${ }^{18,19}$, which suggests that sterol homeostasis is achieved by regulating HMGR at the protein level. ERAD is also involved in regulating HMGR at the protein level in Medicago truncatula ${ }^{12}$ and at the activity level in A. thaliana ${ }^{20}$. In addition to the similar regulations to those of yeast and animals, plants have the potential to evolve a unique regulation ${ }^{21}$. However, plant-specific mechanism underlying sterol biosynthesis is not known.

Lipid bodies (oil bodies) are intracellular structures present in the seed and leaf cells of many land plants. Seed oil bodies function as storage compartments for lipids, while leaf oil bodies function as subcellular factories that have roles in the responses to fungal infection and senescence $^{22,23}$. The main lipids in both seed and leaf oil bodies are triacylglycerols (TAGs), although less is known about the types of lipids accumulated in leaf oil bodies. To better understand leaf oil bodies, we attempted to isolate $A$. thaliana mutants that develop excess oil bodies in the leaves. We found one such mutant, but unexpectedly, an analysis of its oil bodies revealed that they contained high levels of sterol esters in $\sim 1$ - $\mu$ m-diameter structures in the cells. Furthermore, the gene responsible for the mutation was found to have a major role in down-regulating two functional HMGR homologues (HMGR1 and HMGR2) 17, 24, 25.

\section{Results}

\section{hise1 develops lipophilic structures in leaf cells}

To identify oil bodies in leaf cells, we generated a transgenic wild-type $A$. thaliana that expressed a GFP-labeled oil-body membrane-protein (CALEOSIN3, CLO3) ${ }^{22}$ under control of the native $C L O 3$ promoter. $C L O 3$ is expressed at very low levels in mature leaves because 
of their normally low content of oil-bodies, although it is induced by biotic stress and senescence $^{22}$. As expected, CLO3-GFP fluorescence was hardly detected in mature leaves of the transgenic seedlings (Fig. 1a, wild type). We then mutagenized the transgenic plant and screened the mature leaves of the M2 seedlings for mutants with CLO3-GFP fluorescence, which would indicate the presence of oil bodies. In this way, we isolated a recessive mutant that had bright CLO3-GFP fluorescence (Fig. 1a), which, as explained below, we designated hise1-1. hise1-1 had a single mutation at the start codon of the At1g60995 gene, which encodes a transmembrane protein of unknown function (Fig. 1b). Expression of the intact At1g60995 gene in hise1-1 leaves made the CLO3-GFP fluorescence disappear (Fig. 1a), indicating that At1g60995 is responsible for the hise1 phenotype.

We established two recessive mutant alleles (hise1-2 and hise1-3), neither of which had the CLO3-GFP transgene (Fig. 1b). Quantitative PCR analysis revealed that the HiSE1 transcript levels in the hise1-2 and hise1-3 leaves were markedly low (Fig. 1c). The leaves were stained with the lipophilic dye Nile red and inspected with a confocal laser-scanning microscope. Both hise1-2 and hise1-3 had many dot-like Nile-red stainable structures (Fig. 1d). The number of the structures was much higher in all of the hise1 mutant alleles (hise1-1, hise1-2 and hise1-3) than in the wild type (Fig. 1e and Supplementary Fig. 1a). Electron microscopy revealed structures with diameters of $\sim 1 \mu \mathrm{m}$ that were distributed in the cytosol of hise1-2 and hise1-3 leaf cells, but not in the cytosol of wild-type cells (Fig. 1f), indicating that the lipophilic structures are formed in mature leaves as a result of HiSE1 deficiency. To examine the relationship between the lipophilic structures and CLO3-GFP-positive oil bodies, CLO3-GFP was expressed in hise1-2. The lipophilic structures had two populations in both hise1-1 and hise1-2 leaves: a CLO3-GFP-positive population in the leaf marginal areas and a CLO3-GFP-negative population throughout the leaves (Supplementary Fig. 1b,c).

\section{hise1 accumulates high levels of sterol esters in lipophilic structures called SE bodies}

The lipidome of the entire leaf was examined by liquid chromatography-electrospray ionization-mass spectrometry (LC-ESI-MS). hise1 leaves had specific peaks with retention times in the range 8.6 to $9.4 \mathrm{~min}$ (Fig. 2a,b). MS/MS spectra identified all of the hise1specific molecules as sterol esters, each of which was composed of a sterol (stigmasterol, 
cycloartenol/lanosterol, campesterol, 24-methylene-cycloartanol, or sitosterol) and a fatty acid (linolenate or linoleate) (Fig. 2c and Supplementary Figs. 2 and 3). These sterol esters were barely detectable in the wild-type leaves (Fig. 2a,b). On the other hand, hise1 leaves had slightly lower amounts of triacylglycerol (TAG, a major component of seed oil bodies) than the wild-type leaves (Supplementary Fig. 4).

We next isolated the lipophilic structures from hise1 leaves that contained many dot-like Nile-red stainable structures (Fig. 2d). The lipidome of these structures had peaks similar to the hise1-specific peaks on the LC-ESI-MS spectra (Fig. 2e). The lipophilic structures from hise1 leaves predominantly accumulated sterol esters, while the total lipids from hise1 leaves mainly contained phosphatidylcholine and phosphatidylethanolamine in addition to sterol esters (Fig. 2f and Supplementary Data 1). Because of this characteristic feature, we named the mutant as high sterol $\underline{e}$ ster1 (hise1) and the lipophilic structures as sterol-ester bodies (SE bodies).

\section{HiSE1 negatively regulates the protein levels of HMG-CoA reductases}

HiSE1 localized to the ER (see Fig. 6a). To identify its target proteins, we examined proteins in the microsomal fraction from leaves, which includes the ER, by quantitative mass spectrometry. Among 7,000 proteins identified, only two proteins were strikingly different in their levels between the mutant alleles and the wild type (Supplementary Data 2). They were identified as isoforms of HMG-CoA reductase (HMGR) proteins (Fig. 3a), which are ratelimiting enzymes for sterol synthesis on the ER membrane ${ }^{12-16}$. A. thaliana has two HMGR homologues, HMGR1 and HMGR2. hise1-2 had 130-fold higher levels of HMGR1 and 220-fold higher levels of HMGR2 than the wild type, while hise1-3 had 70-fold higher levels of HMGR1 and 140-fold higher levels of HMGR2 (Fig. 3b and Supplementary Table 1). Other enzymes involved in the synthesis of sterols from acetyl CoA (Fig. 3a) were not upregulated in hise1 mutant (Fig. 3b). The hyperaccumulations of the HMGR proteins in hise1-2 and hise1-3 leaves were confirmed by an immunoblot analysis with anti-HMGR antibodies (Fig. 3c). On the other hand, the HMGR1 and HMGR2 mRNA levels were not different between the mutant alleles and the wild type (Fig. 3d). These results indicate that HiSE1 regulates HMGR protein levels, but not HMGR transcript levels. Hence, HiSE1 plays a key 
role in down-regulating HMGR protein levels.

\section{hise1 has remarkably high levels of HMGR activity and sterol-producing activity}

HMGR catalyzes the conversion of HMG-CoA into mevalonate on the ER membrane (Fig. 3a). We measured HMGR activities towards $\left[{ }^{14} \mathrm{C}\right]-\mathrm{HMG}-\mathrm{CoA}$ by using the microsomal fractions from hise1-2 and wild-type leaves (Supplementary Fig. 5a). hise1-2 exhibited $\left[{ }^{14} \mathrm{C}\right]-$ mevalonate-producing activity (Fig. 4a) and the activity of hise1-2 was inhibited by lovastatin, a competitive inhibitor of $\mathrm{HMGR}^{26}$, in a dose-dependent manner (Fig. 4a). The $\left[{ }^{14} \mathrm{C}\right]$-mevalonate production increased with hise1-2 microsomal fraction in an incubation time-dependent manner (Fig. 4b). However, the $\left[{ }^{14} \mathrm{C}\right]$-mevalonate production was hardly detected with the wild-type microsomal fraction (Fig. 4b). To compare specific activities between the wild type and hise1-2, we measured HMGR activities by using crude leaf extracts that had more abundant HMGR proteins than the microsomal fractions (Supplementary Fig. 5b). Specific HMGR activity of hise1-2 was 23-fold higher than that of the wild-type (Fig. 4c). The higher HMGR activity in hise1-2 is consistent with the marked increase in HMGR protein levels in hise1 mutants compared to the wild type (Fig. 3).

To examine the effects of up-regulation of the rate-limiting enzyme HMGR on the sterol biosynthesis pathway, in-vivo productions of squalene (an intermediate) and sterol esters (metabolic end products) were determined by using $\left[{ }^{14} \mathrm{C}\right]$ tracers. In-vivo labeling of leaves with $\left[{ }^{14} \mathrm{C}\right]$-sodium bicarbonate, an initial substrate for carbon fixation, revealed that the level of $\left[{ }^{14} \mathrm{C}\right]$-sterol ester was higher in hise1-2 than in the wild type (Fig. $4 \mathrm{~d}$ ), indicating that carbon fixation products are used preferentially for sterol biosynthesis in hise1. Next, in-vivo labeling with either $\left[2-{ }^{14} \mathrm{C}\right]$-sodium acetate (an acetyl-CoA precursor) or $\left[{ }^{14} \mathrm{C}\right]$-isopentenyl pyrophosphate (IPP, a squalene precursor) showed that $\left[{ }^{14} \mathrm{C}\right]$-sterol esters accumulated to much higher levels in hise1-2 than in the wild type (Fig. 4d), indicating that the hise1-2 mutant has higher levels of sterol-producing activity than the wild type. The production of $\left[{ }^{14} \mathrm{C}\right]$-squalene was also higher in hise1-2 compared to that in the wild type (Fig. 4d). These results indicate that the activity of the sterol biosynthesis pathway was up-regulated in hise1 due to higher HMGR activities. Hence, HiSE1 prevents accumulation of sterols to toxic levels by down-regulating the levels of HMGR proteins. 
Finally, the effect of the HMGR inhibitor lovastatin on plant growth was examined. Inhibiting HMGR proteins stunted the growth of hise1-2, hise1-3, and wild-type seedlings in a dose-dependent manner (0, 100, 300, and $1000 \mathrm{nM})$. However, hise1 mutant alleles were more resistant to the inhibitor than the wild type (Fig. 4e and Supplementary Fig. 6a), in agreement with remarkably higher levels of HMGR proteins (Fig. 3b) and HMGR activities (Fig. 4c) in hise1 than in the wild type. Treatment of seedlings with a competitive inhibitor of squalene synthase (SQS) (zaragozic acid) ${ }^{24}$ also stunted the growth of hise1-2, hise1-3, and wild-type seedlings in a dose-dependent manner (0, 10, and $20 \mu \mathrm{M})$ (Supplementary Fig. $6 b, c)$. The high concentrations of zaragozic acid inhibited the greening of wild-type cotyledons at early developmental stages ( 10-day-old cotyledons) more severely than hise1 cotyledons (Supplementary Fig. 6b,c). These results indicate that hise1 has remarkably high levels of HMGR activity, resulting in up-regulation of sterol biosynthesis pathway.

\section{Detoxification of sterols in hise1}

How do hise1 plants grow normally despite producing potentially toxic levels of sterols? Sterol levels in hise 1 mutant alleles were similar to those in the wild type (Fig. 5e,f), although they had more abundant sterol esters than the wild type (Fig. 2). By focusing on PSAT1, which converts sterols into sterol esters ${ }^{2,3}$, we generated a double-mutant, hise1-3 psat1-2 (Supplementary Fig. 7a). As expected, levels of the sterol esters in the leaves were undetectable, but they completely recovered in hise1-3 psat1-2 by expressing PSAT1 (Fig. 5a and Supplementary Fig. 7b). These results indicate that PSAT1 is responsible for the elevated production of sterol esters in hise1.

To determine whether PSAT1 is involved in SE-body formation in hise1, leaves of hise1-3 psat1-2 and the single mutant psat1 were stained with Nile red. psat1 had no Nile redstainable SE bodies (Fig. 5b), in agreement with the observation that the PSAT1 deficiency failed to produce sterol esters (Fig. 5a) ${ }^{3}$. Similarly, hise1-3 psat1-2 had no SE bodies (Fig. 5b). SE bodies were formed in hise1-3 psat1-2 by expressing PSAT1, but not by expressing HiSE1 (Fig. 5b). A quantitative analysis showed that the ability to form SE bodies (Fig. 5c) was strongly correlated with the levels of sterol ester (Fig. 5a).

hise1-3 psat1-2 seedlings grew very slowly (Fig. 5d), although seedlings of the single 
mutants hise1-2, hise1-3, and psat1-2 grew normally (Fig. 5d and Supplemental Fig. 6). The growth defects in hise1-3 psat1-2 were recovered by expressing either PSAT1 or HiSE1 (Fig. 5d). hise1-3 psat1-2 had 65\% higher levels of total free sterols than the wild type and hise1 (Fig. 5e,f), which may be the reason for its growth defect.

Despite the normal growth of psat1 seedling (Fig. 5d), psat1 exhibits early leaf senescence $^{3}$, possibly due to the higher levels of total free sterols (Fig. 5e,f). In addition to the sterol overaccumulation, sterol deficiency causes defects in plant growth (Fig. 5). These results indicate that sterol levels must be strictly regulated in plants. Sterol homeostasis is essential for not only plant growth but also cellular activity and membrane fluidity ${ }^{1,3,14}$. Taken together, our results show that under HiSE1-deficient conditions, $A$. thaliana plants grow normally with SE bodies and exhibit seedling lethality without them. Thus, HiSE1, by down-regulating HMGR, and PSAT1, by removing sterols, work together to achieve sterol homeostasis.

\section{Localizations of HiSE1 and PSAT1}

To determine the subcellular site of HiSE1, we generated a GFP-fusion of HiSE1 (HiSE1n-GFP-HiSE1c), in which GFP was inserted into the first loop of the membrane protein HiSE1 (Supplementary Fig. 8a), and expressed it in $N$. benthamiana. The GFP fluorescence of HiSE1n-GFP-HiSE1c had a network structure and co-localized with an ER marker (ER-mCherry) (Fig. 6a). No HiSE1n-GFP-HiSE1c signals overlapped with the FM464-labeled plasma membrane (Supplementary Fig. 8b). These results indicate that HiSE1 localizes to the ER membrane.

We next expressed an RFP fusion of PSAT1 (PSAT1-TagRFP) in $N$. benthamiana. The RFP fluorescence revealed punctate structures (Fig. 6b) that were not labeled with markers of five cellular structures (Golgi, mitochondria, trans-Golgi network, endosomes or ER-plasma membrane contact sites) (Supplementary Fig. 9), consistent with a previous report that PSAT1 localized to unknown punctate structures ${ }^{27}$. On the other hand, more than $90 \%$ of PSAT1TagRFP punctate structures were labeled with HiSE1n-GFP-HiSE1c and the ER markers GFP-HDEL and GFP-RHD3 (Fig. 6b,c). These results raise the possibility that the ER microdomain to which PSAT1 localizes is the site where SE bodies are generated. 


\section{Discussion}

Sterol homeostasis is important for plant growth because sterols are essential lipids but high levels of sterols are toxic. In this study, we identified HiSE1 as a key factor in plant sterol homeostasis. We propose a model of a HiSE1-dependent fail-safe regulatory system composed of two processes (Fig. 7). First, HiSE1 negatively regulates the protein levels of the rate-limiting enzyme HMGR on the ER membrane, by which the HMGR activity is kept at the minimum levels to avoid sterol overproduction. Second, if this is not successful, excess sterols are converted to sterol esters on the PSAT1-localized ER microdomains, which are then segregated in the SE bodies. Defects in these processes caused a defect in plant growth (Fig. 5d) due to overaccumulation of sterols. In contrast, plants with sequestered SE bodies grew normally, demonstrating that sequestered SE bodies are nontoxic. Hence, the regulatory system involving HiSE1 and SE bodies provides an efficient and accurate system for plant sterol homeostasis.

The present results clearly show that HiSE1 reduces HMGR1 and HMGR2 protein levels to a bare minimum, but that it does not affect the transcript levels of genes encoding these proteins. How HiSE1 regulates HMGR protein levels is unknown. HiSE1 shares 50\% amino acid sequence similarity (50\% positive substitution) with the mouse ER membrane protein membralin (NP_001346561.1), which down-regulates the multi-subunit protease $\gamma$ secretase through ERAD ${ }^{28,29}$. However, whether membralin is involved in sterol homeostasis is unknown. These results imply that HiSE1 regulates ERAD-mediated down-regulation of HMGR proteins.

In yeast and animals, regulatory factors including E3 ubiquitin ligases function in the ERAD of HMGR proteins ${ }^{21}$. In plants, the E3 ubiquitin ligase MAKIBISHI1 mediates the ERAD of HMGR proteins in Medicago truncatula ${ }^{12}$. Deficiency of MAKIBISHI1 resulted in a 1.5-fold increase in HMGR protein levels compared to control plants ${ }^{12}$. By contrast, deficiency in HiSE1 resulted in the double-digit increase in HMGR protein levels compared to the wild type (Fig. 3b). These data suggest that plants have evolved a unique HiSE1dependent mechanism to regulate HMGR protein levels. 


\section{Methods}

Plant materials and growth conditions. A. thaliana ecotype Columbia (Col-0) was used as the wild type. Three T-DNA mutants were obtained from the Arabidopsis Biological Resource Center at Ohio State University: SALK_013823 (hise1-2), SALK_042821 (hise13), and SALK_117091C (psat1-2). In hise1-2, T-DNA was inserted between 2,773 and 2,774 bp downstream of the start codon of the HiSE1 gene. In hise1-3, T-DNA was inserted between 3,210 and 3,211 bp downstream of the start codon of the HiSE1 gene, although abnormal splicing occurred frequently. The following primer sets were used for genotyping by PCR: 5'-TGGTTCACGTAGTGGGCCATCG-3' for the border primer of T-DNA insertion (LBa1), 5'-TTCAGTGGCTTGAGAGTAAAACT-3' and 5'GGCAGTGAGATTATCACGAGCAA-3' for hise1-2 and hise1-3 (the size of PCR product is 1742 bp), and 5'-ACCCCCTTTTGCATTCATAAC-3' and 5'CTCTCTGGTGTAACGTTTGGC-3' for psat1-2 (the size of PCR product is $1087 \mathrm{bp}$ ). Two double mutants (hise1-2 psat1-2 and hise1-3 psat1-2) were generated by crossing between single mutants. A. thaliana seeds were surface sterilized with $70 \%$ ethanol, dried, sown on Murashige-Skoog (MS) agar plates (Wako, Tokyo, Japan), and incubated at $4^{\circ} \mathrm{C}$ for 3 days to break seed dormancy. The seeds were germinated and grown at $22-25^{\circ} \mathrm{C}$ under continuous light $\left(100 \mu \mathrm{Es}^{-1} \mathrm{~m}^{-2}\right)$ or under a 16-h light $\left(100 \mu \mathrm{Es}^{-1} \mathrm{~m}^{-2}\right) / 8$-h dark photoperiod for 2-3 weeks, followed by growth in vermiculite at $22-25^{\circ} \mathrm{C}$ under a 16 -h light $\left(100 \mu \mathrm{Es}^{-1} \mathrm{~m}^{-2}\right) / 8$-h dark photoperiod. Alternatively, A. thaliana seeds were grown on Rockwool at $23^{\circ} \mathrm{C}$ under a $16-\mathrm{h}$ light $\left(100 \mu \mathrm{Es}^{-1} \mathrm{~m}^{-2}\right) / 8$-h dark photoperiod. The first true leaves (juvenile leaves) of $A$. thaliana were used except as specifically described.

Inhibitor treatments. Seventeen seed grains of each line (the wild type, hise1-2, and hise1-3) were sown on MS agar plates that contained 0, 100, 300 and 1,000 nM lovastatin (Tokyo Chemical Industry Co., Ltd., Tokyo, Japan) and then grown for 2 weeks. The numbers of plants which had opening third true leaves were measured for quantitative analysis. We repeated the experiment three times. Three biological replicates were performed for quantitative analysis. Microsoft excel 2013 was used for statistical tests throughout the statistical analyses in this study.

Fifteen seed grains of each line (the wild type, hise1-2, and hise1-3) were sown on MS agar plates that contained 0, 10, and $20 \mu \mathrm{M}$ zaragozic acid (Funakoshi, Tokyo, Japan) and then grown for 10 days. The numbers of seedlings with senesced cotyledons were counted 
and the ratios to fifteen seedlings of each line were calculated. Three biological replicates were performed for quantitative analysis.

Isolation of the hise1 mutant. Transgenic A. thaliana expressing CLO3-GFP under the control of the native CLO3 promoter (pCLO3::CLO3-GFP) was used as the parent line ${ }^{22}$. The pCLO3::CLO3-GFP seeds were mutagenized by soaking for $16 \mathrm{~h}$ in 0.2 or $0.25 \%(\mathrm{v} / \mathrm{v})$ methanesulfonic acid ethyl ester (Sigma-Aldrich, Tokyo, Japan) and washed for $6 \mathrm{~h}$ in running water to obtain M1 seeds. The M1 seeds were planted, and after self-fertilization, M2 seeds were collected from individual M1 plants to generate the M2 lines. Thirty seeds per M2 line were planted, and 10- to 17-day-old seedlings were collected. Each seedling was examined under a fluorescence microscope or fluorescence stereomicroscope, and a mutant line that exhibited abnormal CLO3-GFP fluorescence was selected; this line was designated high sterol ester1-1 (hise1-1). The hise1-1 mutant was backcrossed once with the wild type containing pCLO3::CLO3-GFP.

Map-based cloning of HiSE1. The hise1-1 homozygous mutant (ecotype Columbia) was crossed with Landsberg erecta wild-type plants to generate the mapping population. In the F2 generation, the hise1-1 mutant was selected and subjected to DNA isolation from leaf tissue. The polymorphism between Columbia and Landsberg erecta was analyzed using a combination of cleaved amplified polymorphic sequence and simple sequence length polymorphism markers ${ }^{30}$ with data obtained from The Arabidopsis Information Resource (http://www.arabidopsis.org). F2 plants showing the hise1 phenotype were screened and used for rough mapping. HiSE1 was localized to chromosome 1. For fine-scale mapping, DNA was isolated from F2 plants. The nucleotide sequences of both DNA strands were determined using an ABI Prism Big Dye Terminator cycle sequence reaction kit (Applied Biosystems, Foster City, CA) and a DNA sequencer (Prism 3100; Applied Biosystems).

Nile red staining. Nile red staining was performed as described previously ${ }^{22}$. A. thaliana leaves were vacuum-infiltrated for $5 \mathrm{~min}$ in Nile red stain in water. The fluorescence of Nile red was examined under a confocal laser-scanning microscope (Fluoview FV500; Olympus, Tokyo, Japan) at a wavelength of $543 \mathrm{~nm}$. Differential interference contrast (DIC) images were taken under the same microscope.

Confocal laser-scanning microscopy. Fluorescent images of transgenic plants were obtained under three confocal laser-scanning microscopes: LSM510 META (Carl Zeiss, Jena, Germany), LSM780 (Carl Zeiss) and Fluoview FV500 (Olympus). The softwares used were ZEN 2.1 (black) of Carl ZEISS and cellSens Dimension (ver 1.16) of Olympus. DIC images 
were obtained using the same microscopes. The procedures used were described previously ${ }^{31}$. The 488-nm laser was used to excite GFP, whereas the 543-nm laser was used to excite RFP, FM4-64 and Nile red. For plasma membrane staining, leaves were exposed to $2 \mu \mathrm{M}$ FM4-64 for 5 min. At least two biological replicates were analyzed.

Quantitative analysis of SE bodies. The number of sterol-ester bodies in the wild type, hise1-2, and hise1-3 was counted in each micrograph $(147.5 \mu \mathrm{m} \times 128.3 \mu \mathrm{m})$, with five micrographs analyzed per line $(n=5)$. The number of leaf oil bodies in wild type, hise1-3, psat1-2, hise1-3 psat1-2, and hise1-3 psat1-2 harboring either p35S::HiSE1 or 35S::PSAT1 was counted in each micrograph $(135 \mu \mathrm{m} \times 135 \mu \mathrm{m})$, with six micrographs analyzed per line $(n=6)$. At least three biological replicates were analyzed.

Lipid analysis. Total lipids were extracted from 2-week-old $A$. thaliana leaves with a mixture of chloroform and methanol, followed by washing with water. Lipidomic analysis of the lipid extract were performed by LC-ESI-MS in the positive ion mode ${ }^{22}$. Lipid species were separated on a C18 column. The hise1-specific lipid species was characterized by MS/MS experiment, using authentic sterol esters (cholesteryl linoleate, cholestery palmitate) as references. Free sterols were analyzed by LC-ESI-MS after picolinoyl derivatization. See Supplementary Methods for more detail.

Vector construction. Vectors were constructed using Gateway Technology (Invitrogen, Carlsbad, CA) with the destination vectors pFAST-G01 $1^{32,33}$ and pFAST-R02 ${ }^{32,33}$, pH2GW7 $7^{32}$ and pB2GW7 $7^{32}$ (Plant System Biology, Gent, Belgium), and pGWB660 ${ }^{34}$ and pGWB406 ${ }^{35}$. The full-length cDNA fragment of Arabidopsis thaliana HiSE1 (HiSE1cFULL), starting from nucleotide 1 (corresponding to A of the start codon ATG) to nucleotide 1,872 (corresponding to A of the stop codon), was amplified by PCR using the primer set: $5^{\prime}$ -

CACCATGGATCCGGAGCAGACGTTTAT-3' (forward) and 5'TTAATCAACTGAAAGAGGATCAT-3' (reverse). The HiSE1cFULL PCR product was cloned into pENTR/D-TOPO (Invitrogen) via TOPO cloning to construct the entry clone, which was then cloned into the destination vectors pFAST-R02 and pGWB406 via LR cloning (Invitrogen), creating two vectors, pFAST-R02-HiSE1cFULL and pGWB406HiSE1cFULL, respectively. The N-terminal fragment of HiSE1 cDNA (HiSE1cN), starting from nucleotide 1 (corresponding to A of the start codon ATG) to nucleotide 549, was PCR amplified using the forward primer 5'-CACCATGGATCCGGAGCAGACGTTTAT-3' and reverse primer 5'-GCCAGATCCGCCATCAAGATTTAGCGGTAT-3', with pGWB406HiSE1cFULL as the template. The C-terminal fragment of HiSE1 cDNA (HiSE1cC), starting 
from nucleotide 550 to nucleotide 1872 (corresponding to A of the stop codon), was PCR amplified using the primer set 5'-TCTGGCTCCGGCGTGCCCAAATTGTTGCAT-3' (forward) and 5'-TTAATCAACTGAAAGAGGATCAT-3' (reverse), with pGWB406HiSE1cFULL as the template. A fragment of the GFP gene, starting from nucleotide 1 (corresponding to A of the start codon ATG) to nucleotide 717, was PCR amplified using the primers 5'-GGCGGATCTGGCATGGTGAGCAAGGGCGAG-3' (forward) and 5'GCCGGAGCCAGACTTGTACAGCTCGTCCAT-3' (reverse), with pFAST-G01 as the template. Full-length HiSE1 cDNA containing a GFP fragment insert (HiSE1cN-GFPHiSE1cC) was PCR amplified from HiSE1cN, HiSE1cC, and GFP fragment using the primer set: 5'-CACCATGGATCCGGAGCAGACGTTTAT-3' (forward) and 5'TTAATCAACTGAAAGAGGATCAT-3' (reverse). The HiSE1cN-GFP-HiSE1cC PCR product was cloned into pENTR/D-TOPO (Invitrogen) via TOPO cloning to produce an entry clone, which was then cloned into the destination vector pB2GW7 via LR cloning (Invitrogen), thus creating the vector pB2GW7-HiSE1cN-GFP-HiSE1cC.

Full-length A. thaliana PSAT1 cDNA (PSAT1cFULL), starting from nucleotide 1 (corresponding to A of the start codon ATG) to nucleotide 1,902 (corresponding to A of the stop codon), was PCR amplified using the primer set: $5^{\prime}$ -

CACCATGGGAGCGAATTCGAAATCAGT-3' (forward) and 5'TTATATGTACTGGAGAAGCATAT-3' (reverse). The PSAT1cFULL PCR product was cloned into pENTR/D-TOPO (Invitrogen) via TOPO cloning to produce an entry clone, which was then cloned into the destination vector pH2GW7 via LR cloning (Invitrogen) to construct the final vector, pH2GW7-PSAT1cFULL.

The full-length fragment of PSAT1 cDNA, except for the stop codon, (PSAT1cFULLstop) from nucleotide 1 (corresponding to A of the start codon ATG) to nucleotide 1,899 was PCR amplified using the primers 5'-CACCATGGGAGCGAATTCGAAATCAGT-3' (forward) and 5'-TATGTACTGGAGAAGCATATTAG-3' (reverse), with pH2GW7PSAT1cFULL as the template. The PSAT1cFULL-stop PCR product was cloned into pENTR/D-TOPO (Invitrogen) via TOPO cloning to produce an entry clone, which was then cloned into the destination vector pGWB660 via LR cloning (Invitrogen) to construct the final vector, pGWB660-PSAT1cFULL-stop.

Quantitative real time PCR analysis. Whole leaves of 2-week-old wild-type, hise1-2 and hise1-3 plants were used. Total RNA was isolated from the leaves using an RNeasy Plant Mini Kit (QIAGEN) and treated with DNase I. cDNA was prepared from the total RNA with 
a reverse transcriptase, ReverTra Ace (Toyobo, Osaka, Japan). The expression levels of mRNA were quantified using a KAPA SYBR FAST Universal Kit (Kapa Biosystems, Wilmington, MA) and Thermal Cycler Dice Real Time System Lite (Takara, Shiga, Japan) with gene-specific-primer sets (5'-AGAGTTGTTGGATATGATACT-3’ and 5'CACCACATTTCGTCACCAAGT-3' for HiSE1, 5'-CCTGACATGGATGTGATTGG-3' and 5'-CAGCAGCAGGTTTCTTGTCC-3' for HMGR1, 5'-CTTCCCGATGGTGACGAC-3' and 5'-TGCTTGTGATGCAAGTTGTG-3' for HMGR2, and 5'CTTGGTGTCAAGCAGATGATTT-3' and 5'-CGTACCTAGCCTTGGAGTATTTG-3’ for EF1a). The relative quantity of target mRNA was normalized using EF1a as a control. Three biological replicates were performed.

Isolation of microsome samples. Whole leaves of 4-week-old wild-type, hise1-2 and hise1-3 plants were used. The leaves $(0.5 \mathrm{~g})$ were chopped with a razor blade in a petri dish on ice in $1.5 \mathrm{~mL}$ of chopping buffer containing $50 \mathrm{mM}$ Hepes-NaOH (pH 7.5), 5 mM EDTA, $0.4 \mathrm{mM}$ sucrose and protease inhibitor cocktail cOmplete (Sigma-Aldrich). The homogenate was filtered through a cell strainer. The filtrate was centrifuged at $1,000 \times g$ for $10 \mathrm{~min}$ at $4^{\circ} \mathrm{C}$. The $1 \mathrm{~mL}$ supernatant was centrifuged at $8,000 \times g$ for $20 \mathrm{~min}$ at $4^{\circ} \mathrm{C}$. The supernatant was ultracentrifuged at $100,000 \times g$ for $60 \mathrm{~min}$ at $4^{\circ} \mathrm{C}$. The pellet was re-suspended in $0.15 \mathrm{~mL}$ of chopping buffer and designated as the microsomal fraction.

Mass spectrometry for quantitative proteomics. The microsome samples were resolved by SDS-PAGE, followed by in-gel digestion using trypsin as described previously ${ }^{36}$. The resulting peptides were measured ( $n=3)$ by Q Exactive hybrid mass spectrometer (Thermo Fisher Scientific, San Jose, CA) essentially as described previously ${ }^{37}$. MS/MS spectra were interpreted and peak lists were generated by Proteome Discoverer 2.2.0.388 (Thermo). Searches were performed by using the SEQUEST against Arabidopsis thaliana (TAIR10) peptide sequence. Searching parameters were set as follows: enzyme selected as used with two maximum missing cleavage sites, a mass tolerance of $10 \mathrm{ppm}$ for peptide tolerance, 0.02 Da for MS/MS tolerance, fixed modification of carbamidomethyl (C), and variable modification of oxidation (M). Peptide identifications were based on significant Xcorr (high confidence filter). Peptide identification and modification information returned from SEQUEST were manually inspected and filtered to obtain confirmed peptide identification and modification lists of HCD MS/MS. Precursor ion intensity (normalized by total peptide amount) was used for label-free quantification.

Immunoblot analysis. A. thaliana leaves were homogenized in 15 volumes (w/v) of SDSsample buffer [100 mM Tris-HCl, pH 6.8, 4\% (w/v) SDS, 20\% (v/v) glycerol, and 10\% (v/v) 
2-mercaptoethanol]. The homogenates $(15 \mu \mathrm{L})$ were subjected to SDS-PAGE on 5-15\% acrylamide gels (Wako). The separated proteins on the gels were transferred electrophoretically to polyvinylidene difluoride membranes (Immobilon-P; Millipore, Billerica, MA). The membranes were treated with blocking solution [ $5 \%$ skim milk, Tris- $\mathrm{HCl}$, $\mathrm{pH} 7.5$, and $0.1 \%(\mathrm{v} / \mathrm{v})$ Triton $\mathrm{X}$ ] and then incubated for $1 \mathrm{hr}$ in anti-HMG1cd antibody ${ }^{38}$ (diluted $1: 1,000$ ) at $25^{\circ} \mathrm{C}$, or for $1 \mathrm{hr}$ in monoclonal anti-Actin (plant) antibody produced in mouse clone 10-B3 (diluted 1: 2,000)(Sigma-Aldrich, St. Louis, MO). Horseradish peroxidase conjugated anti-rabbit IgG (GE Healthcare, Tokyo, Japan) or anti-mouse IgG that were diluted (1:2000) were used as the second antibody. Immunodetection was performed with a chemiluminescent system using ImmunoStar LD (Wako) and C-DiGit blot scanner (LI-COR, Lincoln, NE). The membrane was stained with Coomassie Brilliant Blue (CBB).

HMGR activity. HMGR activity was measured as described previously ${ }^{39,40}$, with a slight modification. Microsomal fractions were prepared from leaves of 4-week-old hise1-2 and wild-type plants. The leaves (2.5 g fresh weight) were chopped with a razor blade in a petri dish on ice in $7.5 \mathrm{~mL}$ of a buffer solution [100 mM potassium phosphate (pH 7.0), $5 \mathrm{mM}$ EDTA, and 0.4 M sucrose]. The homogenate was filtered through gauze and the filtrate was centrifuged at $1,000 \times g$ and $4{ }^{\circ} \mathrm{C}$ for $10 \mathrm{~min}$. The supernatant was centrifuged at 8,000 $\times g$ and $4{ }^{\circ} \mathrm{C}$ for $20 \mathrm{~min}$. The supernatant was ultracentrifuged at $91,000 \times g$ and $4{ }^{\circ} \mathrm{C}$ for $90 \mathrm{~min}$. The pellet was resuspended in $0.5 \mathrm{~mL}$ of $100 \mathrm{mM}$ potassium phosphate buffer ( $\mathrm{pH}$ 7.0) and used as a microsomal fraction. The protein concentration in the resuspensions was measured with TaKaRa BCA Protein Assay Kit (Takara). Then, the protein concentrations of the microsomal fractions were adjusted to $0.3 \mathrm{mg}$ protein $/ \mathrm{mL}$.

Crude extracts were prepared from leaves of 3-week-old hise1-2 and wild-type plants. The leaves ( $0.3 \mathrm{~g}$ fresh weight) were homogenized in $0.9 \mathrm{~mL}$ of $100 \mathrm{mM}$ potassium phosphate (pH 7.0). The homogenate was centrifuged at $8,000 \times g$ and $4^{\circ} \mathrm{C}$ for $10 \mathrm{~min}$. The supernatant was used as a crude extract. The protein concentration in the resuspensions was measured with TaKaRa BCA Protein Assay Kit (Takara). Then, the protein concentrations of crude extracts from the wild type and hise $1-2$ were adjusted to 7.0 and $0.7 \mathrm{mg}$ protein/mL, respectively.

The reaction mixture $(26-\mu \mathrm{L})$ was composed of a microsomal fraction or a crude extract $(18.6 \mu \mathrm{L}), 100 \mathrm{mM}$ potassium phosphate (pH 7.0), $3 \mathrm{mM}$ NADPH, $10 \mathrm{mM}$ dithiothreitol, and $0.03 \mu \mathrm{Ci}\left[3-{ }^{14} \mathrm{C}\right]$-HMG-CoA in the presence $(1,10$ and $100 \mu \mathrm{M})$ or absence of lovastatin. The mixtures were incubated at $30^{\circ} \mathrm{C}$ for 30,60 and $180 \mathrm{~min}$. The reaction was then terminated 
by adding $5 \mu \mathrm{L}$ of mevalonate lactone $(1 \mathrm{mg} / \mathrm{mL})$ and $5 \mu \mathrm{L}$ of $6 \mathrm{~N} \mathrm{HCI}$ followed by incubating for $15 \mathrm{~min}$ at room temperature. After adding $125 \mu \mathrm{L}$ of $1 \mathrm{M}$ potassium phosphate (pH 6.0) and $300 \mu \mathrm{L}$ of ethyl acetate, the solution was vortexed and centrifuged for separating into two layers. The upper layer fraction (lipid fraction) $(260 \mu \mathrm{L})$ containing $\left[{ }^{14} \mathrm{C}\right]$-mevalonate was collected. A part of the fraction was applied to INSTA-GEL (PerkinElmer) to measure the radioactivity. The rest part was subjected to silica gel thin layer chromatography (TLC) by using acetone:venzene $(1: 1, \mathrm{v} / \mathrm{v})$. The TLC plate was exposed to an imaging plate (BAS-IP SR 2040, Fujifilm) overnight. Signals on the TLC plate were detected with a scanner (Typhoon FLA7000, GE Healthcare) and analyzed with Image Quant TL (GE Healthcare) to quantify the radioactivity of produced $\left[{ }^{14} \mathrm{C}\right]$-mevalonate. Three biological replicates were analyzed.

Metabolism of labeled compounds. Whole leaves of 4-week-old hise1-2 and wild-type plants were used. Leaf segments ( $5 \mathrm{~mm} \times 5 \mathrm{~mm}$; ca.156 mg) were incubated in a $30 \mathrm{~mL}$ Erlenmeyer flask fitted with a glass tube, containing a piece of folded filter paper impregnated with $0.1 \mathrm{~mL} 20 \%(\mathrm{w} / \mathrm{w}) \mathrm{KOH}$ in the center well, with $37 \mathrm{kBq}$ of $\left[4-{ }^{14} \mathrm{C}\right]$-isopentenyl pyrophosphate (1.872GBq mmol ${ }^{-1}$; Perkin Elmer), $74 \mathrm{kBq}$ of $\left[{ }^{14} \mathrm{C}\right]$-sodium bicarbonate $(2.18$ GBq $\mathrm{mmol}^{-1}$, Perkin Elmer), or $74 \mathrm{kBq}$ of $\left[2-{ }^{14} \mathrm{C}\right]$-acetic acid sodium salt (2.15 $\mathrm{GBq} \mathrm{mmol}^{-1}$; GE Healthcare) for $24 \mathrm{~h}$ under illumination $\left(76.5 \mu \mathrm{mol} \mathrm{m}^{-2} \mathrm{~s}^{-1}\right)$ at $25^{\circ} \mathrm{C}$. After incubation, a $0.5 \mathrm{~mL}$ aliquot of supernatant was mixed with $0.5 \mathrm{~mL}$ of $0.1 \mathrm{~N} \mathrm{HCl}$ in a $30 \mathrm{~mL}$ Erlenmeyer flask fitted with a glass tube, containing a piece of folded filter paper impregnated with 0.1 $\mathrm{mL} 20 \%(\mathrm{w} / \mathrm{w}) \mathrm{KOH}$ in the center well. Carbon dioxide $\left(\mathrm{CO}_{2}\right)$ dissolved in the medium was captured by the $\mathrm{KOH}$-impregnated filter paper. Total lipids were extracted according to the method of Bligh \& Dyer ${ }^{41}$. The upper layer, lower layer, and residue were referred to as hydrophilic, hydrophobic, and starch fractions, respectively. The glass tube and filter paper from the center well were transferred to a $50 \mathrm{~mL}$ flask containing $10 \mathrm{~mL}$ distilled water. Radioactive signal of a $0.5 \mathrm{~mL}$ aliquot was determined using the liquid scintillation counter to estimate the amount of ${ }^{14} \mathrm{CO}_{2}$ released during metabolism. Radioactivity in the hydrophilic and starch fractions was also measured with the liquid scintillation counter. Hydrophobic fraction was separated by silica gel thin layer chromatography (TLC). Squalene was separated using hexane:diethyl ether:acetic acid solution (80:20:1, v/v/v) or hexane, and quantified based on the comparison of its $\mathrm{R}_{\mathrm{f}}$ value with that of a standard. The hydrophobic fraction was applied to a TLC plate, exposed to an imaging plate (BAS-IP SR 2040, Fujifilm) overnight, detected with a scanner (Typhoon FLA7000, GE Healthcare), and analyzed with Image Quant TL (GE Healthcare). Radioactivity was quantified based on a standard curve generated from 
known amounts of $\left[2-{ }^{14} \mathrm{C}\right]$-acetic acid sodium salt. Radioactivities incorporated into squalene and sterol esters are given based on the fresh weights of leaves. Three biological replicates were performed.

Stable transformation of $\boldsymbol{A}$. thaliana plants. The vector pBGWF7-pCLO3-CLO3 ${ }^{22}$ or pFAST-R02-HiSE1cFULL was introduced into hise1-2 or hise1-1 mutant plants, respectively, via Agrobacterium-mediated transformation (strain GV3101) using the floral dip method ${ }^{42}$, thus generating transgenic hise1-2 and hise1-1 plants containing the pCLO3::CLO3-GFP or p35S::HiSE1 vector, respectively. Similarly, vector pFAST-R02-HiSE1cFULL or pH2GW7PSAT1cFULL was introduced into hise1-3 psat1-2 double mutants, as described above, to generate transgenic hise1-3 psat1-2 plants containing p35S::HiSE1 or p35S::PSAT1 vector, respectively.

Fluorescence microscopy analysis. Fluorescent images of leaves of wild-type, hise1-1, and hise1-2 plants were obtained with a fluorescence microscope (BX53; Olympus) equipped with a CCD camera (DP74; Olympus). A mirror unit (U-FBNA, Olympus) was used for GFP and Nile red fluorescence. Two biological replicates were performed. The number of SE bodies in wild-type, hise1-1, and hise1-2 leaves was counted in each micrograph (300 $\mu \mathrm{m} \times$ $185 \mu \mathrm{m})$, and six micrographs were analyzed per line $(n=6)$. A mirror unit U-FGNA (Olympus) was used for HMGR1-RFP fluorescence. Three biological replicates were performed.

Reverse transcription PCR (RT-PCR). Total RNA was isolated from A. thaliana seedlings using RNeasy Plant Mini Kit (Qiagen). The RNA was treated with DNase I and used for cDNA synthesis using Ready-To-Go RT-PCR Beads (GE Healthcare), followed by PCR using the following primer sets: 5'-TTCAGTGGCTTGAGAGTAAAACT-3' (forward) and 5'ATCAACTGAAAGAGGATCATGAT-3' (reverse) for HiSE1, 5'CTCTCTGGTGTAACGTTTGGC-3' (forward) and 5'-TATGTACTGGAGAAGCATATTAG3'(reverse) for PSAT1, and 5'-ACTGGAGGTTTTGAGGCTGGTAT-3' (forward) and 5'GCACCGTTCCAATACCACCAATC-3' (reverse) for EF1a. The size of PCR products of HiSE1, PSAT1, and EF1a was 1,268 (for both the wild type and hise1-3), 1,164, and 494 bp, respectively. At least three biological replicates were performed for all RT-PCR analyses. DNA sequencing of the transcripts revealed that hise1-3 had at least three types of sequences; the wild-type sequence, a sequence with a 5-bp insertion at the beginning of the 11th exon, and a sequence that contains the 9th intron, suggesting that abnormal splicing occurs in hise13. 
Transient expression assay in Nicotiana benthamiana. Vectors including pB2GW7HiSE1cN-GFP-HiSE1cC, pGWB660-PSAT1cFULL-stop, pBIC/ER-mCherry ${ }^{43}$, pBI121/p35S:SP-GFP-HDEL ${ }^{44}$, pHGW/proRHD3::GFP-RHD3 ${ }^{45}$, pGWB2/ST-Venus ${ }^{46}$, mt$\mathrm{gk}^{47}$, and $\mathrm{pGWB1/GFP-SYP61}{ }^{48,49}$ were used for transient expression assays, as described previously ${ }^{50}$. Unpublished vectors were also used, including pGWB5/ARA6-GFP provided by Dr. Emi Ito at International Christian University and Dr. Kazuo Ebine at the National Institute for Basic Biology, pGWB2/GFP-ARA7 provided by Dr. Tatsuaki Goh at Kobe University, and pSYT1-GFP provided by Dr. Kazuo Ebine. These vectors were independently transformed into A. tumefaciens strain GV3101. Transformed A. tumefaciens cells were inoculated into leaves of 4-7-week-old $N$. benthamiana plants. Two days after inoculation, leaves were observed under a confocal laser scanning microscope. Percentages of PSAT1TagRFP punctate structures, labeled with each organelle marker, were calculated. At least two biological replicates of microscope analysis were performed.

Isolation of SE bodies. SE bodies were isolated as described previously ${ }^{51}$, with a slight modification. Briefly, aerial parts (3.5 g) of juvenile leaves of 4-week-old hise1-2 plants were homogenized in $5 \mathrm{~mL}$ of $20 \%$ sucrose in a buffer (100 mM Tris-HCl [pH 7.5], $10 \mathrm{mM} \mathrm{KCl}$, and 1 mM EDTA); this buffer was used throughout the experiment. The suspension was centrifuged at $10,000 \times g$ for $10 \mathrm{~min}$. A $2 \mathrm{~mL}$ floating lipid fraction was collected and centrifuged at $20,000 \times g$ for $10 \mathrm{~min}$. A $0.6 \mathrm{~mL}$ volume of top-most fraction was collected and mixed with $0.5 \mathrm{~mL}$ of $20 \%$ sucrose. The suspension was covered with $0.2 \mathrm{~mL}$ of $15 \%$ sucrose, followed by $0.4 \mathrm{~mL}$ of $5 \%$ sucrose, and then centrifuged at 20,000 $\times g$ for $10 \mathrm{~min}$. After removing the bottom-most fraction, the remaining sample was mixed with $0.5 \mathrm{~mL}$ of $20 \%$ sucrose. The suspension was covered with $0.2 \mathrm{~mL}$ of $15 \%$ sucrose, followed by $0.4 \mathrm{~mL}$ of $5 \%$ sucrose, and then centrifuged at $20,000 \times g$ for $10 \mathrm{~min}$. The bottom-most fraction was removed, and the remaining sample was mixed with $0.3 \mathrm{~mL}$ of $20 \%$ sucrose. The suspension was covered with $0.4 \mathrm{~mL}$ buffer and centrifuged at $20,000 \times g$ for $10 \mathrm{~min}$. Subsequently, the bottom-most fraction was removed, and the remaining sample was mixed with $0.5 \mathrm{~mL}$ buffer. The suspension was centrifuged at 20,000 $\times g$ for $10 \mathrm{~min}$, and the floating lipid fraction was stored at $-80^{\circ} \mathrm{C}$ until further use.

Chemicals. Cholesterol-25,26,26,26,27,27,27- $d_{7}$ (99 atom \% D), stigmastanol, and brassicasterol were purchased from C/D/N Isotopes (Quebec, Canada), Wako Pure Chemicals (Osaka, Japan), and Larodan Fine Chemicals (Solna, Sweden), respectively. $\beta$-Sitosterol, campesterol, and stigmasterol were obtained from Tama Biochemicals (Tokyo, Japan). Cholesterol, cycloartenol, cholesteryl linoleate, cholestery palmitate, and 1,2-didecanoyl-sn- 
glycero-3-phosphocholine were obtained from Sigma-Aldrich (St. Louis, MO). Picolinic acid, 4-dimethylaminopyridine, and 2-methyl-6-nitrobenzoic anhydride were purchased from Tokyo Chemical Industry (Tokyo, Japan).

Lipidomic analysis. Crude lipids were extracted according to the method of Bligh and Dyer $^{41}$, with a slight modification ${ }^{52,39,40}$. Briefly, fresh deuterium-labeled cholesterol was added to the extraction solvent as an internal standard for free sterol analysis. A. thaliana leaves (average fresh weight $=30 \mathrm{mg}$ ) were harvested, placed in a microcentrifuge tube along with a zirconia bead, and immediately frozen in liquid nitrogen. Samples were homogenized under cryogenic conditions using a BMS Shake Master at $900 \mathrm{rpm}$ for $2 \mathrm{~min}$. To the frozen plant powder, 20-volumes of $\mathrm{CHCl}_{3}: \mathrm{MeOH}$ : water mixture (50:100:31.45, v/v/v), containing 1 $\mu \mathrm{M}$ 1,2-didecanoyl-sn-glycero-3-phosphocholine and $2.5 \mu \mathrm{M}$ cholesterol$25,26,26,26,27,27,27-d_{7}$, was added and vortexed. Samples were centrifuged at $10,000 \times g$ for $5 \mathrm{~min}$ at room temperature. The chloroform layer $(200 \mu \mathrm{l})$ was collected in a new microcentrifuge tube, and water $(52.6 \mu \mathrm{l})$ was added. After vigorous mixing, the sample was incubated on ice for $10 \mathrm{~min}$, and then centrifuged at $1,000 \times g$ for $3 \mathrm{~min}$ at $4^{\circ} \mathrm{C}$. After centrifugation, $85 \mu \mathrm{l}$ of the lower layer was collected in a new microcentrifuge tube. The organic solvent was removed using a centrifugal concentrator, and the residue was dissolved in $162 \mu \mathrm{l}$ ethanol. After centrifugation at $10,000 \times g$ for $15 \mathrm{~min}$ at $4^{\circ} \mathrm{C}$, the supernatant was collected and subjected to liquid chromatography-mass spectrometry (LC-MS) analysis on a Waters Xevo G2 Qtof mass spectrometer as described ${ }^{22}$, except that the conditions for recording mass spectra were changed to the following: scan range, mass-to-charge ratio $(\mathrm{m} / \mathrm{z})$ 100 to 2,000; polarity, positive ion mode; capillary voltage, $3 \mathrm{kV}$; cone voltage, $20 \mathrm{~V}$; source temperature, $120^{\circ} \mathrm{C}$; desolvation temperature, $450^{\circ} \mathrm{C}$; cone gas flow, $50 \mathrm{~L} \mathrm{~h}^{-1}$; desolvation gas flow, $600 \mathrm{~L} \mathrm{~h}^{-1}$; and nebulizer gas, $\mathrm{N}_{2}$. Tandem mass spectrometry (MS/MS) data were recorded in positive ion mode using collision energy ramp mode (ramp start, $20 \mathrm{~V}$; ramp end, $40 \mathrm{~V}$ ), with the other parameters as described above. The recorded data were analyzed as described previously ${ }^{53}$.

Free sterols were analyzed via LC-MS after picolinoyl derivatization, as described by Yamashita et al. (2007) ${ }^{54}$. Samples for lipidomic analysis were diluted 10-fold in ethanol, and $0.1 \mathrm{~mL}$ aliquots were dried in a centrifugal concentrator. Dried lipid extracts were combined with a freshly prepared mixture of derivatization reagents containing $0.05 \mathrm{~mL}$ each of picolinic acid (90 mg), 4-dimethylaminopyridine (30 mg), and 2-methyl-6-nitrobenzoic anhydride $(150 \mathrm{mg})$ dissolved in dry tetrahydrofuran $(3 \mathrm{~mL})$ and triethylamine $(0.6 \mathrm{~mL})$. The resulting mixture was vortexed and incubated for $30 \mathrm{~min}$ at room temperature. Subsequently, 
water $(1 \mathrm{~mL})$ and ethyl acetate $(0.4 \mathrm{~mL})$ were added to each sample and thoroughly mixed. Samples were then centrifuged at 3,000 $\times g$ for $5 \mathrm{~min}$. The upper layer was collected, dried, and dissolved in ethanol $(0.2 \mathrm{~mL})$. After centrifugation at $10,000 \times g$ for $10 \mathrm{~min}$, the supernatant was collected and analyzed on a Shimadzu LCMS-8050 equipped with a Shimadzu Nexera ultra high performance liquid chromatography (UHPLC) system. Chromatographic separation was performed at a flow rate of $0.4 \mathrm{~mL} \mathrm{~min}^{-1}$ on an ODS column (Kinetex $2.6 \mu \mathrm{m} \mathrm{C18} 100 \AA$, $30 \mathrm{~mm} \times 2.1 \mathrm{~mm}$; Phenomenex, CA) at 55 ${ }^{\circ} \mathrm{C}$ using solvents A (acetonitrile:water:formic acid = 200:800:1, v/v/v) and B (acetonitrile:2-propanol:formic acid $=100: 900: 1, \mathrm{v} / \mathrm{v} / \mathrm{v})$. Gradients of both solvents were the same as those used for general lipidomic analysis ${ }^{55}$. Picolinoyl esters of free sterols were detected via selected reaction monitoring (SRM) in the positive ion mode. The SRM transitions for each sterol derivative were as follows: cholesterol- $d_{7}(\mathrm{~m} / \mathrm{z} 499.40>376.35, \mathrm{CE}-15)$, cholesterol $(\mathrm{m} / \mathrm{z} 492.4>$ 369.35, CE -15), $\beta$-sitosterol ( $\mathrm{m} / \mathrm{z} 520.40>397.40, \mathrm{CE}-15)$, campesterol $(\mathrm{m} / \mathrm{z} 506.40>$ 383.35, CE -15), stigmastanol ( $\mathrm{m} / \mathrm{z} 522.45>399.40, \mathrm{CE}-15)$, brassicasterol $(\mathrm{m} / \mathrm{z} 504.40>$ 381.35, CE -15), stigmasterol ( $\mathrm{m} / \mathrm{z} 518.40>395.35$, CE - 15), cycloartenol $(\mathrm{m} / \mathrm{z} 532.40>$ 409.40, CE -15), and 24-methylenecycloartanol ( $\mathrm{m} / \mathrm{z}$ 546.45 > 423.40, CE - 15).

Lipidome analysis of the lipid fraction prepared from the isolated SE bodies was performed as described above.

\section{Data availability}

Sequence data from this study can be found in the GenBank/EMBL data libraries under the following accession numbers: CLO3 (At2g33380), HiSE1 (At1g60995), PSAT1 (At1g04010), HMGR1 (At1g76490), HMGR2 (At2g17370) and EF1a (At5g60390). The data that support the findings of this study are available from the corresponding author upon request. 


\section{References}

1. Ferrer, A., Altabella, T., Arro, M. \& Boronat, A. Emerging roles for conjugated sterols in plants. Prog. Lipid. Res. 67, 27-37 (2017).

2. Banas, A. et al. Cellular sterol ester synthesis in plants is performed by an enzyme (phospholipid:sterol acyltransferase) different from the yeast and mammalian acylCoA:sterol acyltransferases. J. Biol. Chem. 280, 34626-34634 (2005).

3. Bouvier-Nave, P. et al. Involvement of the phospholipid sterol acyltransferase1 in plant sterol homeostasis and leaf senescence. Plant Physiol. 152, 107-119 (2010).

4. Schaller, H. et al. Expression of the Hevea brasiliensis (H.B.K.) Mull. Arg. 3Hydroxy-3-Methylglutaryl-Coenzyme A Reductase 1 in Tobacco Results in Sterol Overproduction. Plant Physiol. 109, 761-770 (1995).

5. Maillot-Vernier, P., Gondet, L., Schaller, H., Benveniste, P. \& Belliard, G. Genetic study and further biochemical characterization of a tobacco mutant that overproduces sterols. Mol. Gen. Genet. 231, 33-40 (1991).

6. Gondet, L., Bronner, R. \& Benveniste, P. Regulation of Sterol Content in Membranes by Subcellular Compartmentation of Steryl-Esters Accumulating in a SterolOverproducing Tobacco Mutant. Plant Physiol. 105, 509-518 (1994).

7. Wilkinson, S.C., Powls, R. \& Goad, L.J. The effects of excess exogenous mevalonic acid on sterol and steryl ester biosynthesis in celery (Apium graveolens) cell suspension cultures. Phytochemistry 37, 1031-1035 (1994).

8. Diener, A.C. et al. Sterol methyltransferase 1 controls the level of cholesterol in plants. Plant Cell 12, 853-870 (2000).

9. Sonawane, P.D. et al. Plant cholesterol biosynthetic pathway overlaps with phytosterol metabolism. Nat. Plants 3, 16205 (2016).

10. Liu, J. \& Nes, W.D. Steroidal triterpenes: design of substrate-based inhibitors of ergosterol and sitosterol synthesis. Molecules 14, 4690-4706 (2009).

11. Burg, J.S. \& Espenshade, P.J. Regulation of HMG-CoA reductase in mammals and yeast. Prog. Lipid. Res. 50, 403-410 (2011).

12. Pollier, J. et al. The protein quality control system manages plant defence compound synthesis. Nature 504, 148-152 (2013).

13. Chye, M.L., Tan, C.T. \& Chua, N.H. Three genes encode 3-hydroxy-3-methylglutarylcoenzyme A reductase in Hevea brasiliensis: hmg1 and hmg3 are differentially expressed. Plant Mol. Biol. 19, 473-484 (1992).

14. Suzuki, M. et al. Loss of function of 3-hydroxy-3-methylglutaryl coenzyme A reductase 1 (HMG1) in Arabidopsis leads to dwarfing, early senescence and male sterility, and reduced sterol levels. Plant J. 37, 750-761 (2004).

15. Ohyama, K., Suzuki, M., Masuda, K., Yoshida, S. \& Muranaka, T. Chemical phenotypes of the hmg1 and hmg2 mutants of Arabidopsis demonstrate the in-planta role of HMG-CoA reductase in triterpene biosynthesis. Chem. Pharm. Bull. (Tokyo) 55, 1518-1521 (2007).

16. Ferrero, S. et al. Proliferation and Morphogenesis of the Endoplasmic Reticulum 
Driven by the Membrane Domain of 3-Hydroxy-3-Methylglutaryl Coenzyme A Reductase in Plant Cells. Plant Physiol. 168, 899-914 (2015).

17. Learned, R.M. \& Fink, G.R. 3-Hydroxy-3-methylglutaryl-coenzyme A reductase from Arabidopsis thaliana is structurally distinct from the yeast and animal enzymes. Proc. Natl. Acad. Sci. U. S. A. 86, 2779-2783 (1989).

18. Gardner, R.G., Shearer, A.G. \& Hampton, R.Y. In vivo action of the HRD ubiquitin ligase complex: mechanisms of endoplasmic reticulum quality control and sterol regulation. Mol. Cell Biol. 21, 4276-4291 (2001).

19. Faulkner, R.A., Nguyen, A.D., Jo, Y. \& DeBose-Boyd, R.A. Lipid-regulated degradation of HMG-CoA reductase and Insig-1 through distinct mechanisms in insect cells. J. Lipid Res. 54, 1011-1022 (2013).

20. Doblas, V.G. et al. The SUD1 gene encodes a putative E3 ubiquitin ligase and is a positive regulator of 3-hydroxy-3-methylglutaryl coenzyme a reductase activity in Arabidopsis. Plant Cell 25, 728-743 (2013).

21. Erffelinck, M.L. \& Goossens, A. Review: Endoplasmic Reticulum-Associated Degradation (ERAD)-Dependent Control of (Tri)terpenoid Metabolism in Plants. Planta Med. 84, 874-880 (2018).

22. Shimada, T.L. et al. Leaf oil body functions as a subcellular factory for the production of a phytoalexin in Arabidopsis. Plant Physiol. 164, 105-118 (2014).

23. Shimada, T.L., Takano, Y. \& Hara-Nishimura, I. Oil body-mediated defense against fungi: From tissues to ecology. Plant Signal. Behav. 10, e989036 (2015).

24. Caelles, C., Ferrer, A., Balcells, L., Hegardt, F.G. \& Boronat, A. Isolation and structural characterization of a cDNA encoding Arabidopsis thaliana 3-hydroxy-3methylglutaryl coenzyme A reductase. Plant Mol. Biol. 13, 627-638 (1989).

25. Enjuto, M. et al. Arabidopsis thaliana contains two differentially expressed 3-hydroxy3-methylglutaryl-CoA reductase genes, which encode microsomal forms of the enzyme. Proc. Natl. Acad. Sci. U. S. A. 91, 927-931 (1994).

26. Kobayashi, K. et al. Lovastatin insensitive 1, a Novel pentatricopeptide repeat protein, is a potential regulatory factor of isoprenoid biosynthesis in Arabidopsis. Plant Cell Physiol. 48, 322-331 (2007).

27. Kopischke, M. et al. Impaired sterol ester synthesis alters the response of Arabidopsis thaliana to Phytophthora infestans. Plant J. 73, 456-468 (2013).

28. Yang, B. et al. The critical role of membralin in postnatal motor neuron survival and disease. Elife 4 (2015).

29. Zhu, B. et al. ER-associated degradation regulates Alzheimer's amyloid pathology and memory function by modulating gamma-secretase activity. Nat. Commun. 8, 1472 (2017).

30. Tamura, K., Shimada, T., Kondo, M., Nishimura, M. \& Hara-Nishimura, I. KATAMARI1/MURUS3 Is a novel golgi membrane protein that is required for endomembrane organization in Arabidopsis. Plant Cell 17, 1764-1776 (2005).

31. Shimada, T.L., Shimada, T., Takahashi, H., Fukao, Y. \& Hara-Nishimura, I. A novel role for oleosins in freezing tolerance of oilseeds in Arabidopsis thaliana. Plant J. 55, 798-809 (2008). 
32. Karimi, M., Inze, D. \& Depicker, A. GATEWAY vectors for Agrobacterium-mediated plant transformation. Trends Plant. Sci. 7, 193-195 (2002).

33. Shimada, T.L., Shimada, T. \& Hara-Nishimura, I. A rapid and non-destructive screenable marker, FAST, for identifying transformed seeds of Arabidopsis thaliana. Plant J. 61, 519-528 (2010).

34. Nakamura, S. et al. Gateway binary vectors with the bialaphos resistance gene, bar, as a selection marker for plant transformation. Biosci. Biotechnol. Biochem. 74, 13151319 (2010).

35. Nakagawa, T. et al. Improved Gateway binary vectors: high-performance vectors for creation of fusion constructs in transgenic analysis of plants. Biosci. Biotechnol. Biochem. 71, 2095-2100 (2007).

36. Fujimoto, S., Sugano, S.S., Kuwata, K., Osakabe, K. \& Matsunaga, S. Visualization of specific repetitive genomic sequences with fluorescent TALEs in Arabidopsis thaliana. J. Exp. Bot. 67, 6101-6110 (2016).

37. Shishido, Y. et al. A covalent G-site inhibitor for glutathione S-transferase Pi (GSTP11). Chem. Commun. (Camb) 53, 11138-11141 (2017).

38. Tang, J., Kobayashi, K., Suzuki, M., Matsumoto, S. \& Muranaka, T. The mitochondrial PPR protein LOVASTATIN INSENSITIVE 1 plays regulatory roles in cytosolic and plastidial isoprenoid biosynthesis through RNA editing. Plant J. 61, 456-466 (2010).

39. Maurey, K., Wolf, F. \& Golbeck, J. 3-Hydroxy-3-Methylglutaryl Coenzyme A Reductase Activity in Ochromonas malhamensis: A System to Study the Relationship between Enzyme Activity and Rate of Steroid Biosynthesis. Plant Physiol. 82, 523527 (1986).

40. Bach, T.J., Rogers, D.H. \& Rudney, H. Detergent-solubilization, purification, and characterization of membrane-bound 3-hydroxy-3-methylglutaryl-coenzyme A reductase from radish seedlings. Eur. J. Biochem. 154, 103-111 (1986).

41. Bligh, E.G. \& Dyer, W.J. A rapid method of total lipid extraction and purification. Can. J. Biochem. Physiol. 37, 911-917 (1959).

42. Bechtold, N. \& Pelletier, G. In planta Agrobacterium-mediated transformation of adult Arabidopsis thaliana plants by vacuum infiltration. Methods Mol. Biol. 82, 259-266 (1998).

43. Kaido, M., Funatsu, N., Tsuno, Y., Mise, K. \& Okuno, T. Viral cell-to-cell movement requires formation of cortical punctate structures containing Red clover necrotic mosaic virus movement protein. Virology 413, 205-215 (2011).

44. Mitsuhashi, N., Shimada, T., Mano, S., Nishimura, M. \& Hara-Nishimura, I. Characterization of organelles in the vacuolar-sorting pathway by visualization with GFP in tobacco BY-2 cells. Plant Cell Physiol. 41, 993-1001 (2000).

45. Ueda, H. et al. Phosphorylation of the C Terminus of RHD3 Has a Critical Role in Homotypic ER Membrane Fusion in Arabidopsis. Plant Physiol. 170, 867-880 (2016).

46. Uemura, T. et al. Qa-SNAREs localized to the trans-Golgi network regulate multiple transport pathways and extracellular disease resistance in plants. Proc. Natl. Acad. Sci. U. S. A. 109, 1784-1789 (2012). 
47. Nelson, B.K., Cai, X. \& Nebenfuhr, A. A multicolored set of in vivo organelle markers for co-localization studies in Arabidopsis and other plants. Plant J. 51, 11261136 (2007).

48. Uemura, T. et al. Systematic analysis of SNARE molecules in Arabidopsis: dissection of the post-Golgi network in plant cells. Cell Struct. Funct. 29, 49-65 (2004).

49. Choi, S.W. et al. RABA members act in distinct steps of subcellular trafficking of the FLAGELLIN SENSING2 receptor. Plant Cell 25, 1174-1187 (2013).

50. Sparkes, I.A., Runions, J., Kearns, A. \& Hawes, C. Rapid, transient expression of fluorescent fusion proteins in tobacco plants and generation of stably transformed plants. Nat. Protoc. 1, 2019-2025 (2006).

51. Brocard, L. et al. Proteomic Analysis of Lipid Droplets from Arabidopsis Aging Leaves Brings New Insight into Their Biogenesis and Functions. Front. Plant Sci. 8, 894 (2017).

52. Okazaki, Y. et al. A new class of plant lipid is essential for protection against phosphorus depletion. Nat. Commun. 4, 1510 (2013).

53. Okazaki, Y. \& Saito, K. Plant Lipidomics Using UPLC-QTOF-MS. Methods Mol. Biol. 1778, 157-169 (2018).

54. Yamashita, K. et al. Use of novel picolinoyl derivatization for simultaneous quantification of six corticosteroids by liquid chromatography-electrospray ionization tandem mass spectrometry. J. Chromatogr. A. 1173, 120-128 (2007).

55. Okazaki, Y. et al. Induced accumulation of glucuronosyldiacylglycerol in tomato and soybean under phosphorus deprivation. Physiol. Plant. 155, 33-42 (2015).

\section{Corresponding author}

Correspondence should be addressed to I. H.-N. (e-mail: ihnishi@gr.bot.kyoto-u.ac.jp).

\section{Acknowledgments}

We are grateful to Tsuyoshi Nakagawa (Shimane University), Sumie Ishiguro (Nagoya University), Masanori Kaido (Kyoto University), Tomohiro Uemura (University of Tokyo), Andreas Nebenführ (University of Tennessee), Emi Ito (International Christian University), Kazuo Ebine (National Institute for Basic Biology), Tatsuaki Goh (Nara Institute of Science and Technology), and Plant System Biology (VIB) for their donations of the vectors; to Saki Arai (Ochanomizu University), Rei Iwahori (Ochanomizu University), Kouji Takano (RIKEN Center for Sustainable Resource Science) for their technical assistance; to the Arabidopsis Biological Resource Center for providing seeds of $A$. thaliana T-DNA insertion mutants; to the Model Plant Research Facility (NIBB BioResource Center) and the Japan Advanced Plant Science Network (Riken) for their technical supports; to Toshiya Muranaka (Osaka University) for anti-HMG1cd antibody; to Mitsumasa Hanaoka (Chiba University) for real time PCR analysis; and James Raymond (Eigoken) for critical readings of this manuscript. This work was supported by Grants-in-Aid for Scientific Research to I.H.-N. (nos. 15 H05776 
and 22000014) and to T.L.S. (nos. 16K18834 and 19K05809) from the Japan Society for the Promotion of Science (JSPS), by Leading Initiative for Excellent Young Researchers (LEADER) to T.L.S. (no. J16HJ00026) from the Ministry of Education, Culture, Sports, Science and Technology in Japan (MEXT), by SUNBOR GRANT of Suntory Foundation for life science to T.L.S., by Kato Memorial Bioscience Foundation to T.L.S., by Phytochemical Plant Molecular Science of Strategic Priority Research Promotion Program to T.L.S. and K.S. from Chiba University, and by the Hirao Taro Foundation of KONAN GAKUEN for Academic Research to I.H.-N.

\section{Author contributions}

T.L.S., T.S., and I.H.-N. designed the research. T.L.S. performed the experiments except for lipidome, proteome, and radiolabeling experiments. Y.O., Y.H. and K.S. performed lipidome experiments, K.K. performed proteome experiments, and K.O. and M.K. performed in vivo and in vitro radiolabeling experiments. H.U. contributed to cell biological analysis, and A.N., T.U. and Y.T. contributed to discussion. T.L.S. and I.H.-N. analyzed whole data and wrote the article.

\section{Additional information}

Supplementary information is available online. Reprints and permissions information is available online at www.nature.com/reprints. Correspondence and requests for materials should be addressed to I.H.N.

\section{Competing interests}

The authors declare no competing financial or non-financial interests. 


\section{FIGURE LEGENDS}

Figure 1 | An A. thaliana mutant, hise1, abnormally develops lipophilic structures. a, GFP fluorescence images and bright field images of 12-day-old plants of wild type, hise1-1, and hise1-1 rescued by expressing p35S::HiSE1. pCLO3::CLO3-GFP was introduced into all the lines. Three biological replicates were performed with similar results. b, Schematic representation of HiSE1 gene (At1g60995) and the positions of the hise1-1 mutation and each T-DNA insertion in two hise1 mutant alleles (hise1-2 and hise1-3). Closed boxes, exons; solid lines, introns; arrows 1 and 2, primers used for genotyping; arrows 1 and 3, primers used for reverse transcription PCR; arrows 4 and 5, primers used for quantitative real time PCR. Schematic representation of HiSE1 protein composed of 623 amino acids. Gray boxes, transmembrane domains. c, The expression levels of HiSE1 mRNA in wild type, hise1-2 and hise 1-3 by quantitative real time PCR analysis. The averages of three biological replicates are shown. Error bars show standard deviations $(n=3)$. Different letters exhibit significant differences ( $p<0.05$, Tukey's test). The intensity value of wild type was defined as 1.0. d, Confocal laser-scanning microscopic images showing lipophilic structures (SE bodies, arrowheads) in juvenile leaves of 2-week-old plant of wild type, hise1-2, and hise1-3. e, A quantitative analysis of SE-body densities in cross sections of confocal laser-scanning microscopic images of juvenile leaves of 2-week-old plants of wild type, hise1-2, and hise1-3. The averages of five biological replicates are shown. Error bars show standard deviations $(n=$ 5). Different letters exhibit significant differences ( $p<0.05$, Tukey's test). f, Electron micrographs showing SE bodies in hise1-2 and hise1-3 juvenile leaves (asterisks). Note that the wild-type leaves hardly develop SE bodies. Ten ultrathin sections of each genotype were inspected with similar results. CW, cell wall.

\section{Figure 2 | hise1 mutant accumulates much higher levels of sterol esters in SE bodies. a,} Liquid chromatography-electrospray ionization-mass spectrometry (LC-ESI-MS) patterns of lipids from juvenile-leaf extracts of wild type, hise1-1 and hise1-2. The intensity of the maximum peak of wild type is $100 \%$. Six biological replicates were performed with similar results. $\mathbf{b}$, These panels show magnified views of the boxed areas of a. c, Identification of hise1-specific sterol esters. Identified sterol esters from hise1-1-specific peaks with retention times in the range 8.6 to $9.4 \mathrm{~min}$ on LC-ESI-MS in $\mathbf{b}$. Annotation shows sterol moiety based on the $\mathrm{m} / \mathrm{z}$ value of fragment ion detected in MS/MS spectra and literature data on phytosterols. Since there are many isomers in phytosterols, some minor SE species are dually annotated. There is also a possibility that sterol moiety should be other isomer of same molecular weight (e.g. the moiety annotated as 24-methylenecycloartanol might be 24methylcycloartenol. They are isomers). Formula shows $[\mathrm{M}+\mathrm{NH} 4]^{+}$ion. d, Bright-field and Nile red-fluorescence images of the SE bodies isolated from the hise1-2 leaves. Three biological replicates were performed with similar results. e, LC-ESI-MS patterns of lipids of 
the isolated SE bodies of hise1-2, total lipids from juvenile-leaf extracts of hise1-3 and wild type. Blank background pattern is shown. The intensity of the maximum peak of wild type is $100 \%$. Three biological replicates were performed with similar results. f, Relative contents of sterol esters, TAGs, phosphatidylcholines (PCs), and phosphatidylethanolamines (PEs). See Supplementary Data 1 for detailed information on the molecular species identified.

Figure 3 | Hyperaccumulation of HMGR proteins in hise1 mutant. a, A schematic view of biosynthesis of sterols via mevalonate pathway, isoprenoid pathway and sterol biosynthetic pathway. The enzymes indicated were detected by quantitative proteomics (see $\mathbf{b}$ and Supplementary Table 1). IPP, isopentenyl pyrophosphate; DMAPP, dimethylallyl pyrophosphate; FPP, farnesyl pyrophosphate. Lovastatin is a competitive inhibitor of HMGR and zaragozic acid is a competitive inhibitor of squalene synthase (SQS). b, Mass spectrometry for quantitative proteomics showing precursor ion intensities of enzymes that were detected in the microsomal fractions from wild type, hise1-2 and hise1-3 leaves. The precursor ion intensities were normalized by total peptide amounts. The intensity value of wild type was defined as 1.0. The averages of three biological replicates are shown. Error bars show standard deviations $(n=3)$. Asterisks show significant differences between wild type and hise1 mutants (two-sided t-test, $\mathrm{p}<0.01$ ). c, An immunoblot analysis of leaf extracts of wild type, hise1-2 and hise1-3 with anti-HMGR and anti-actin antibodies. Three biological replicates were performed with similar results. d, The expression levels of HMGR1 and HMGR2 mRNAs in wild type, hise1-2 and hise1-3 by quantitative real time PCR analysis. The averages of three biological replicates are shown. Error bars show standard deviations ( $n$ $=3$ ). The intensity value of wild type was defined as 1.0. Significant differences in the relative expression levels of HMGR1 and HMGR2 were examined ( $p>0.05$, Tukey's test).

\section{Figure 4 | hise1 mutant has dramatically higher sterol-producing activity than the wild} type. a, Thin-layer chromatography showing $\left[{ }^{14} \mathrm{C}\right]$-mevalonate-producing activity from $\left[{ }^{14} \mathrm{C}\right]-$ HMG-CoA of microsomal fractions of the wild-type and hise1-2 leaves in the presence or absence of a HMGR inhibitor (lovastatin) for $60 \mathrm{~min}$ Two biological replicates were performed with similar results. $\mathbf{b}$, Time-course data for $\left[{ }^{14} \mathrm{C}\right]$-mevalonate production with the wild-type and hise1-2 microsomal fractions (5.6 $\mu \mathrm{g}$ protein). The averages of three biological replicates are shown. Error bars show standard deviations $(n=3)$. A significant difference between the wild type and hise1-2 was determined ( $p=3.6 \times 10^{-6}$, two-sided t-test). c, Specific HMGR activities of crude extracts and microsomal fractions from the wild-type and hise1-2 leaves are shown. Three biological replicates were performed. Data represent average \pm standard deviation $(n=3)$. A significant difference between the wild type and hise1-2 was determined ( $p=5.1 \times 10^{-10}$, two-sided t-test). d, In-vivo labeling with a radioactive $\left[{ }^{14} \mathrm{C}\right]$ - 
sodium bicarbonate as an initial substrate for carbon fixation, $\left[2-{ }^{14} \mathrm{C}\right]$-sodium acetate as an acetyl-CoA precursor, and $\left[{ }^{14} \mathrm{C}\right]-\mathrm{IPP}$ as a squalene precursor. The incorporated radioactivity levels into squalene or sterol esters in the same fresh weights of wild-type and hise1-2 leaves are shown. The averages of three biological replicates are shown. Error bars show standard deviations $(n=3)$. Asterisks exhibit a significant difference between wild type and hise1-2 ( $p$ $<0.05$, two-sided t-test). e, Effects of lovastatin on the ratios of plants with opening third true leaves to 17 plants of wild type, hise1-2 and hise1-3. See Supplementary Fig. 6a for their plant growth. The averages of three biological replicates are shown. Error bars show standard deviations $(n=3)$. Asterisks exhibit a significant difference between wild type and hise1 mutants ( $p<0.05$, two-sided t-test).

\section{Figure 5 | Deficiency of PSAT1 causes defects in SE-body formation and plant growth of} hise1. The wild type, two single mutants (hise1-3 and psat1-2), the double mutant (hise1-3 psat1-2), and the double mutant rescued by expressing either p35S::HiSE1 or p35S::PSAT1 were used. a, The contents of sitosteryl linoleate and campesteryl linoleate in 2-week-old leaves are shown as relative values of signal intensities of $[\mathrm{M}+\mathrm{NH} 4]^{+}$species of sterol esters to that of $[\mathrm{M}+\mathrm{H}]^{+}$of internal standard (1,2-didecanoyl-sn-glycero-3-phosphocholine). The averages of six biological replicates are shown. Error bars show standard deviations $(n=6)$. Different letters exhibit significant differences ( $p<0.05$, Tukey's test). $\mathbf{b}$, Confocal laserscanning microscopic images of leaf cells stained with Nile red. Arrowheads show SE bodies. Three biological replicates were performed with similar results. c, A quantitative analysis of SE-body densities in 2-week-old leaves. The averages of six biological replicates are shown. Error bars show standard deviations $(n=6)$. Different letters exhibit significant differences ( $p<0.05$, Tukey's test). d, Growth phenotypes of 2-week-old seedlings of each line on rock wools. Three biological replicates were performed with similar results. e, Total contents of free sterols in 2-week-old juvenile leaves. Values are given in $\mu \mathrm{g} / \mathrm{g}$ fresh weight (FW). A dotted line shows the total free sterol level of the wild type. The averages of biological replicates with error bars (standard deviations). Wild type $(n=6)$, hise1-3 $(n=6)$, psat1-2 ( $n$ $=5)$, hise1-3 psat1-2 $(n=6)$ and p35S::PSAT1 in hise1-3 psat1-2 $(n=6)$, and p35S::HiSE1 in hise1-3 psat1-2 $(n=5)$. Different letters exhibit significant differences $(p<0.05$, TukeyKramer method). f, Free sterol contents of each line. Sterol moiety was annotated based on the $\mathrm{m} / \mathrm{z}$ value of fragment ion detected in MS/MS spectra and literature data on phytosterols. Data represent average \pm standard deviation. Biological replicates were performed; wild type $(n=6)$, hise1-3 $(n=6)$, psat1-2 $(n=5)$, hise1-3 psat1-2 $(n=6)$ and p35S::PSAT1 in hise1-3 psat1-2 $(n=6)$, and p35S::HiSE1 in hise1-3 psat1-2 $(n=5)$.

Figure 6 | HiSE1 localizes to the ER, while PSAT1 localizes to the ER microdomains. a, 
Transient expression of HiSE1n-GFP-HiSE1c with the ER marker ER-mCherry in $N$. benthamiana leaves. b, Transient expression of PSAT1-TagRFP with each of HiSE1n-GFPHiSE1c, the ER marker GFP-HDEL, and the ER-membrane marker GFP-RHD3 in $N$. benthamiana leaves. c, Labeling rates of PSAT1 punctate structures with the markers indicated. PM, Plasma membrane. The averages with error bars (standard deviations) of five biological replicates are shown. In each experiment, 50 to 100 PSAT1 punctate structures were analyzed. Different letters exhibit significant differences ( $p<0.05$, Tukey's test).

\section{Figure 7 | A hypothetical model of a HiSE1-depedent fail-safe regulatory system for} sterol homeostasis via HMGR down-regulation. The regulatory system is composed of two processes: 1) HiSE1-mediated down-regulation of HMGR to prevent sterol overproduction on the ER and 2) Detoxification of excess amounts of sterols on the PSAT1-localized ER microdomains and segregation of sterol esters into SE bodies. MVA, mevalonate. 


\section{SUPPLEMENTARY FIGURE LEGENDS}

\section{Supplementary Figure 1 | CLO3-GFP-positive and CLO3-GFP-negative populations of} SE bodies in hise1-1 and hise1-2 leaves. a, Quantitative analysis of SE body densities in fluorescence microscopic images of juvenile leaves of 2-week-old wild-type, hise1-1, and hise1-2 plants containing the CLO3-GFP transgene. The averages of six biological replicates are shown. Error bars represent standard deviation $(n=6)$. Different letters indicate significant differences ( $p<0.05$; Tukey's test). b, GFP fluorescence and bright field images of the first true leaves of 2-week-old hise1-1 and hise1-2 plants containing the CLO3-GFP transgene. Three biological replicates were performed with similar results. c, Confocal laserscanning microscopic images showing CLO3-GFP and Nile red-labeled SE bodies of the first true leaves in $\mathbf{b}$. Note that SE bodies are separated into two populations; a CLO3-GFPpositive population in the leaf marginal areas and a CLO3-GFP-negative population throughout the leaves. Three biological replicates were performed with similar results.

\section{Supplementary Figure 2 | Structural characterization of sterol esters via tandem mass} spectrometry (MS/MS) in the positive ion mode. MS/MS spectra of two standard sterol esters and a major hise1-specific sterol ester are shown. The $m / z$ value of the precursor ion, $\left[\mathrm{M}+\mathrm{NH}_{4}\right]^{+}$, was subjected to MS/MS analysis. A fragment ion attributable to sterol moiety formed by the neutral loss of fatty acid moiety was observed as a base peak in the MS/MS spectrum. Three biological replicates were performed with similar results.

\section{Supplementary Figure 3 | MS/MS spectra of a series of sterol esters accumulated} abundantly in hise1 plants. MS/MS spectra of hise1-specific sterol esters are shown. The $\mathrm{m} / \mathrm{z}$ value of the precursor ion, $\left[\mathrm{M}+\mathrm{NH}_{4}\right]^{+}$, was subjected to $\mathrm{MS} / \mathrm{MS}$ analysis. A fragment ion attributable to sterol moiety formed by the neutral loss of fatty acid moiety was observed as a base peak in the MS/MS spectrum. Three biological replicates were performed with similar results.

\section{Supplementary Figure 4 | Liquid chromatography-mass spectrometry (LC-MS) analysis} of triacylglycerols (TAGs) isolated from the juvenile leaves of two hise1 mutants and the wild type. Contents of TAGs are expressed as signal intensities of $[\mathrm{M}+\mathrm{NH} 4]^{+}$species relative to that of $[\mathrm{M}+\mathrm{H}]^{+}$of the internal standard (1,2-didecanoyl-sn-glycero-3-phosphocholine). For example, TAG_52:5 has 52 carbons and 5 double bonds. The averages of six biological replicates are shown. Error bars represent standard deviation $(n=6)$. Different letters indicate significant differences ( $p<0.05$; Tukey's test). 


\section{Supplementary Figure 5 | Microsomal fractions and crude extracts from the wild type} and hise1-2 used for measuring HMGR activities. a, An immunoblot of microsomal fractions of the wild type and hise1-2 with anti-HMGR antibody. The blotting membrane was stained with Coomassie Brilliant Blue (CBB). Three biological replicates were performed with similar results. $\mathbf{b}$, An immunoblot of crude leaf extracts of the wild type and hise1-2 with either anti-HMGR antibody or anti-actin antibody. An asterisk shows a non-specific band. Three biological replicates were performed with similar results.

\section{Supplementary Figure 6 | Effects of the HMGR inhibitor lovastatin and the SQS} inhibitor zaragozic acid on growth of the wild-type and hise1 plants. a, Two-week-old plants of the wild type and hise1 mutant alleles (hise1-2 and hise1-3) on the agar plates in the absence and presence of the HMGR inhibitor lovastatin. White arrowheads show plants that were unable to open the third true leaves. Three biological replicates were performed with similar results. b, Ten-day-old seedlings of the wild type and hise1 mutant alleles on the agar plates in the absence and presence of the SQS inhibitor zaragozic acid. White arrowheads show senesced cotyledons. Three biological replicates were performed with similar results. c, Effects of zaragozic acid on the ratio of seedlings with greening cotyledons to 15 seedlings of the wild type, hise1-2 and hise1-3. See Supplementary Fig. 6b for their plant growth. The averages of three biological replicates are shown. Error bars show standard deviations $(n=3)$. Asterisks show significant differences between wild type and hise 1 mutants ( $p<0.05$, twosided t-test).

\section{Supplementary Figure 7 | Sterol ester contents in leaves of $A$. thaliana mutant lines and} the wild type. a, RT-PCR of HiSE1, PSAT1, and EF1a (control) transcripts in 2-week-old seedlings of each line indicated. Numbers of PCR cycles for each gene are shown. Three biological replicates were performed with similar results. b, Sterol ester contents in juvenile leaves of each line. Signal intensities of $[\mathrm{M}+\mathrm{NH} 4]^{+}$species of sterol esters relative to that of $[\mathrm{M}+\mathrm{H}]^{+}$of the internal standard (1,2-didecanoyl-sn-glycero-3-phosphocholine) are shown. Data represent average \pm standard deviation. Six biological replicates were performed. n.d., not detected.

\section{Supplementary Figure 8 | No overlap of HiSE1n-GFP-HiSE1c signals with the FM4-64-} labeled plasma membrane. a, A schematic view of the GFP fusion, HiSE1n-GFP-HiSE1c. The GFP fragment was inserted between the 183th and 184th amino acid of HiSE1. b, $N$. benthamiana leaves expressing HiSE1n-GFP-HiSE1c were stained with FM4-64 to visualize the plasma membrane (PM). Fluorescence images of the cortical region (upper) and middle region (lower) of the cell are shown. Three biological replicates were performed with similar results. 
Supplementary Figure 9 | Localization of PSAT1 to the endoplasmic reticulum (ER) microdomains. The PSAT1-TagRFP fusion was transiently co-expressed with each organelle marker in $N$. benthamiana leaves. Different organelle markers used included the Golgi marker ST-Venus, the mitochondrial marker mt-gk, the ER-plasma membrane-contact site marker SYT1-GFP, the trans-Golgi-network (TGN) marker GFP-SYP61, and two endosome markers ARA6-GFP and GFP-ARA7. Three biological replicates were performed with similar results.

Supplementary Table 1 | Protein levels of sterol biosynthetic enzymes in microsomal fractions of leaves of wild type, hise1-2 and hise1-3.

Supplementary Data 1 | LC-ESI-MS data showing relative contents of sterol esters, TAGs, phosphatidylcholines (PCs), and phosphatidylethanolamines (PEs) in hise1 mutant leaf extract and the isolated SE bodies.

Supplementary Data 2 | Quantitative proteomics of microsomal fractions from leaves of wild type, hise1-2 and hise1-3. 


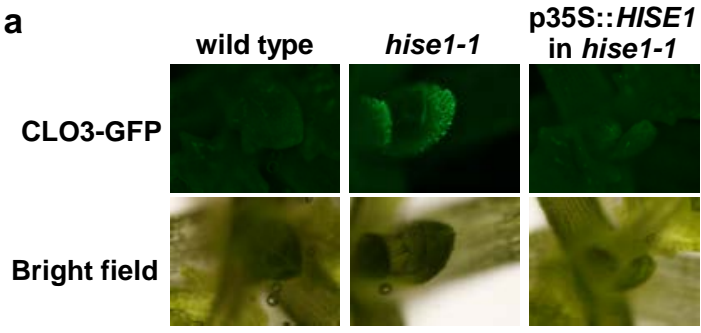

b

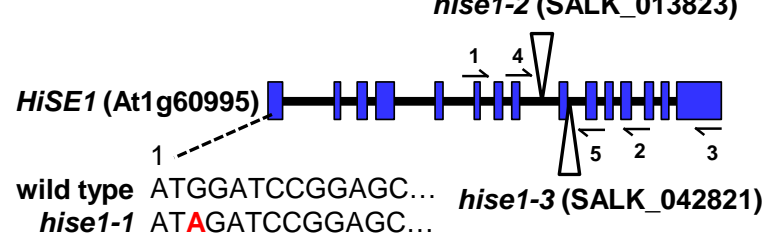

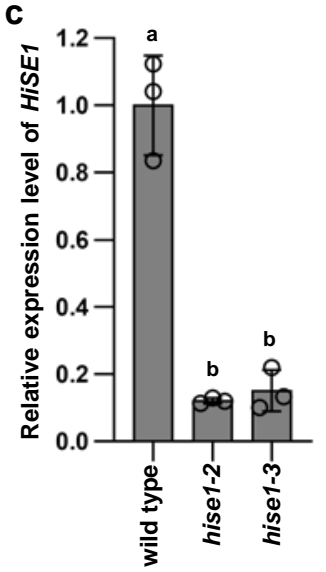
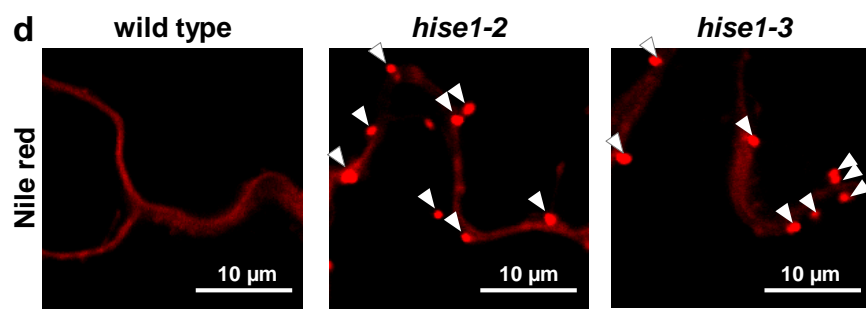

$\mathbf{f}$

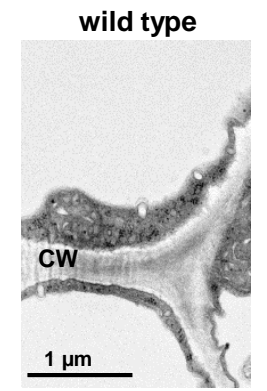

hise1-2

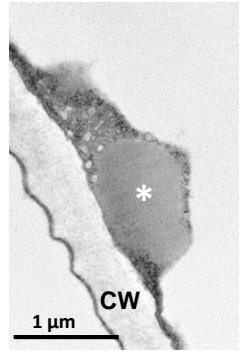

hise1-3

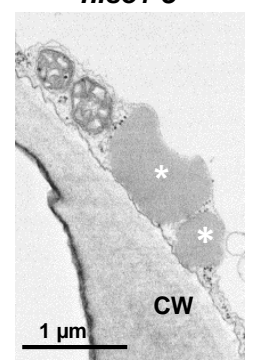

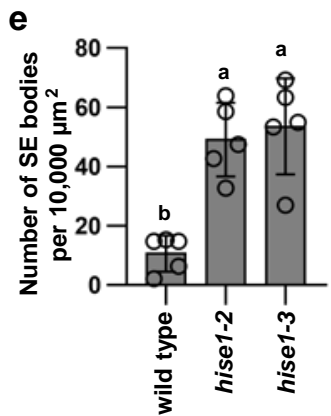

Figure 1 | An A. thaliana mutant, hise1, abnormally develops lipophilic structures. a, GFP fluorescence images and bright field images of 12-day-old plants of wild type, hise1-1, and hise1-1 rescued by expressing p35S::HiSE1. pCLO3::CLO3-GFP was introduced into all the lines. Three biological replicates were performed with similar results. b, Schematic representation of HiSE1 gene (At1g60995) and the positions of the hise1-1 mutation and each T-DNA insertion in two hise1 mutant alleles (hise1-2 and hise1-3). Closed boxes, exons; solid lines, introns; arrows 1 and 2, primers used for genotyping; arrows 1 and 3, primers used for reverse transcription PCR; arrows 4 and 5, primers used for quantitative real time PCR. Schematic representation of HiSE1 protein composed of 623 amino acids. Gray boxes, transmembrane domains. c, The expression levels of HiSE1 mRNA in wild type, hise12 and hise 1-3 by quantitative real time PCR analysis. The averages of three biological replicates are shown. Error bars show standard deviations $(n=3)$. Different letters exhibit significant differences ( $p<0.05$, Tukey's test). The intensity value of wild type was defined as 1.0. d, Confocal laser-scanning microscopic images showing lipophilic structures (SE bodies, arrowheads) in juvenile leaves of 2-week-old plant of wild type, hise1-2, and hise1-3. e, A quantitative analysis of SE-body densities in cross sections of confocal laser-scanning microscopic images of juvenile leaves of 2-week-old plants of wild type, hise1-2, and hise1-3. The averages of five biological replicates are shown. Error bars show standard deviations $(n=5)$. Different letters exhibit significant differences ( $p<0.05$, Tukey's test). f, Electron micrographs showing SE bodies in hise1-2 and hise1-3 juvenile leaves (asterisks). Note that the wild-type leaves hardly develop SE bodies. Ten ultrathin sections of each genotype were inspected with similar results. CW, cell wall. 

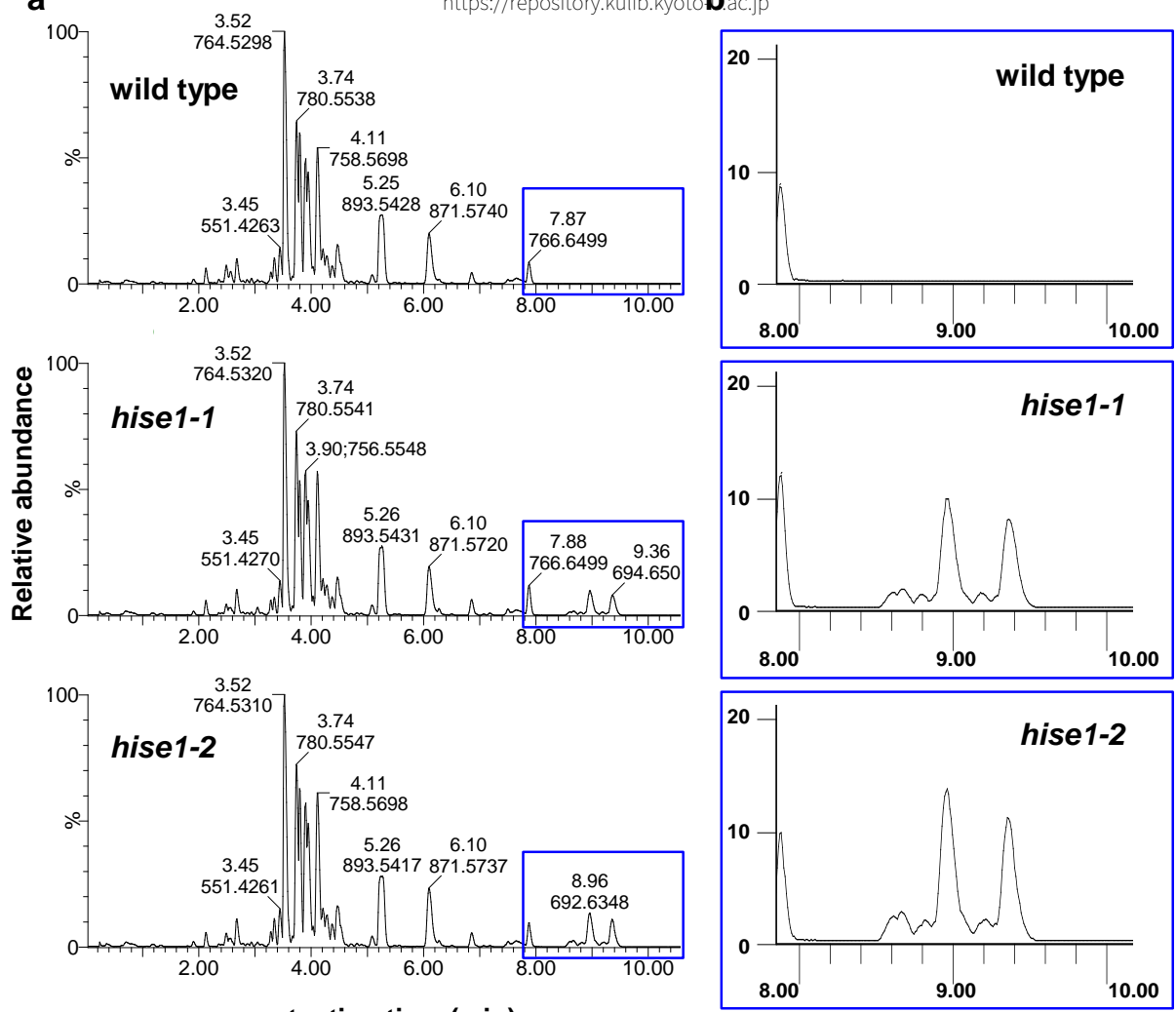

retention time $(\min )$

retention time $(\mathrm{min})$

\begin{tabular}{ccll}
\hline Retention time (min) & $\mathrm{m} / \mathrm{z}$ & \multicolumn{1}{c}{ Annotation } & Formula \\
\hline 8.62 & 690.618 & stigmasteryl linolenate $\left(\left[\mathrm{M}+\mathrm{NH}_{4}\right]^{+}\right)$ & C47H80NO2 \\
8.67 & 704.633 & cycloartenyl/lanosteryl linolenate $\left(\left[\mathrm{M}+\mathrm{NH}_{4}\right]^{+}\right)$ & C48H82NO2 \\
8.80 & 678.618 & campesteryl linolenate $\left(\left[\mathrm{M}+\mathrm{NH}_{4}\right]^{+}\right)$ & C46H80NO2 \\
8.84 & 704.633 & cycloartenyl/lanosteryl linolenate $\left(\left[\mathrm{M}+\mathrm{NH}_{4}\right]^{+}\right)$ & C48H82NO2 \\
8.89 & 718.649 & 24-methylenecycloartanyl linolenate $\left(\left[\mathrm{M}+\mathrm{NH}_{4}\right]^{+}\right)$ & C49H84NO2 \\
8.96 & 692.634 & sitosteryl linolenate $\left(\left[\mathrm{M}+\mathrm{NH}_{4}\right]^{+}\right)$ & C47H82NO2 \\
9.04 & 706.649 & cycloartenyl/lanosteryl linoleate $\left(\left[\mathrm{M}+\mathrm{NH}_{4}\right]^{+}\right)$ & C48H84NO2 \\
9.19 & 680.634 & campesteryl linoleate $\left(\left[\mathrm{M}+\mathrm{NH}_{4}\right]^{+}\right)$ & C46H82NO2 \\
9.23 & 706.649 & cycloartenyl/lanosteryl linoleate $\left(\left[\mathrm{M}+\mathrm{NH}_{4}\right]^{+}\right)$ & C48H84NO2 \\
9.29 & 720.665 & 24-methylenecycloartanyl linoleate $\left(\left[\mathrm{M}+\mathrm{NH}_{4}\right]^{+}\right)$ & $\mathrm{C} 49 \mathrm{H} 86 \mathrm{NO} 2$ \\
9.37 & 694.650 & sitosteryl linoleate $\left.\left([\mathrm{M}+\mathrm{NH}]_{4}\right]^{+}\right)$ & C47H84NO2 \\
\hline
\end{tabular}

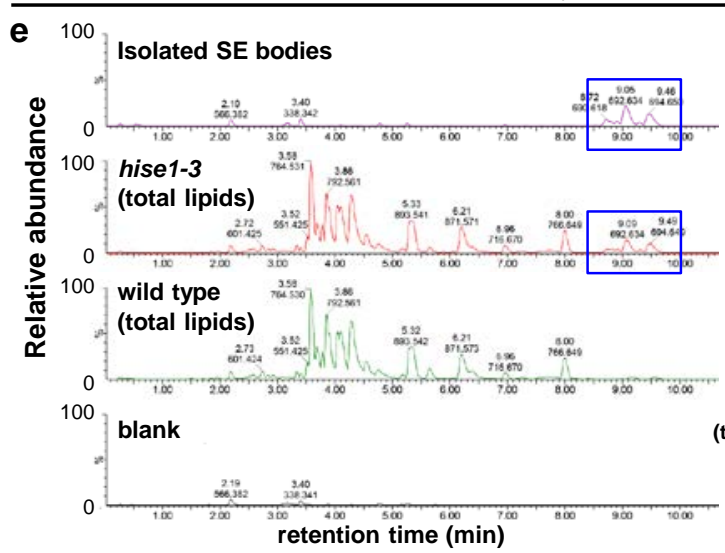

\section{d} Isolated SE bodies from hise1 leaves
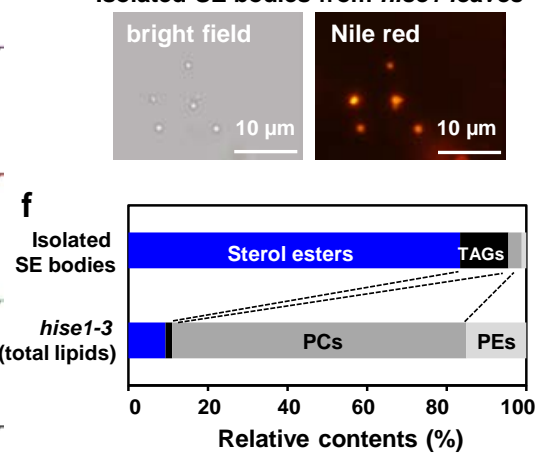

Figure 2 | hise1 mutant accumulates much higher levels of sterol esters in SE bodies. a, Liquid chromatography-electrospray ionization-mass spectrometry (LC-ESI-MS) patterns of lipids from juvenile-leaf extracts of wild type, hise1-1 and hise1-2. The intensity of the maximum peak of wild type is $100 \%$. Six biological replicates were performed with similar results. b, These panels show magnified views of the boxed areas of a. c, Identification of hise1-specific sterol esters. Identified sterol esters from hise1-1-specific peaks with retention times in the range 8.6 to 9.4 min on LCESI-MS in b. Annotation shows sterol moiety based on the $\mathrm{m} / \mathrm{z}$ value of fragment ion detected in MS/MS spectra and literature data on phytosterols. Since there are many isomers in phytosterols, some minor SE species are dually annotated. There is also a possibility that sterol moiety should be other isomer of same molecular weight (e.g. the moiety annotated as 24-methylenecycloartanol might be 24-methylcycloartenol. They are isomers). Formula shows $[\mathrm{M}+\mathrm{NH} 4]^{+}$ion. d, Bright-field and Nile red-fluorescence images of the SE bodies isolated from the hise1-2 leaves. Three biological replicates were performed with similar results. e, LC-ESI-MS patterns of lipids of the isolated SE bodies of hise1-2, total lipids from juvenile-leaf extracts of hise1-3 and wild type. Blank background pattern is shown. The intensity of the maximum peak of wild type is $100 \%$. Three biological replicates were performed with similar results. f, Relative contents of sterol esters, TAGs, phosphatidylcholines (PCs), and phosphatidylethanolamines (PEs). See Supplementary Data 1 for detailed information on the molecular species identified. 
a

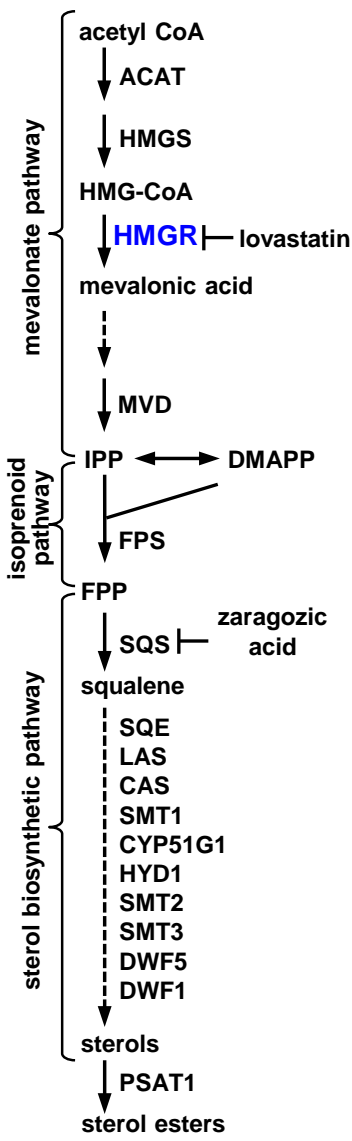

Precursor ion intensity

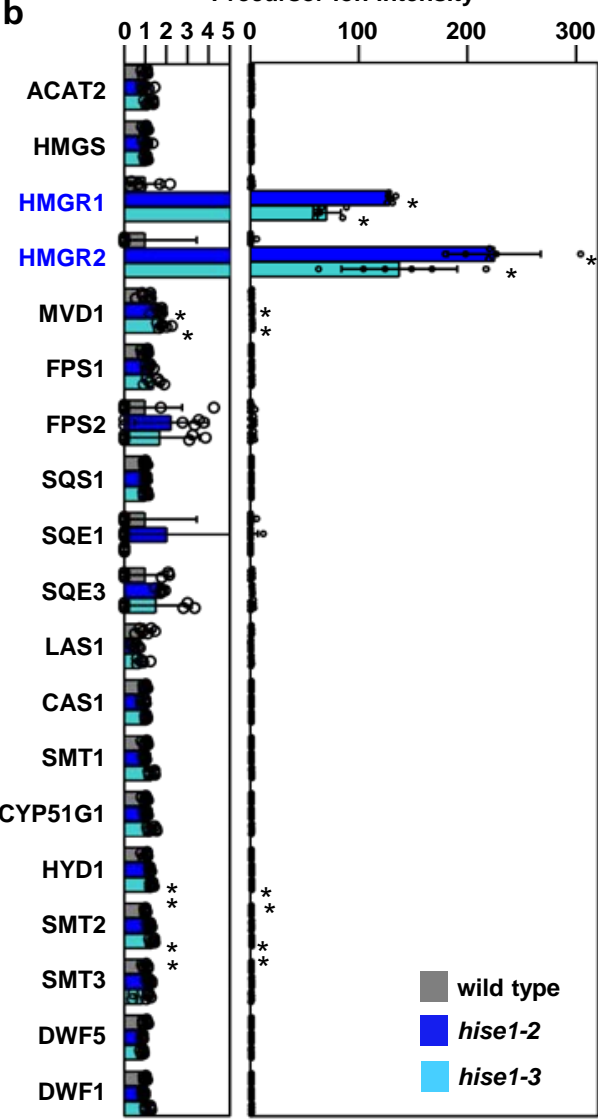

C

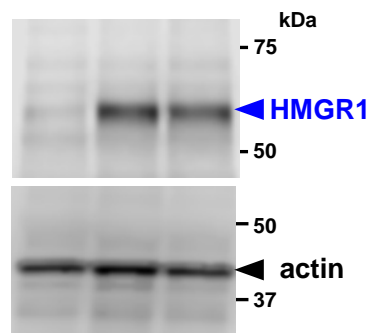

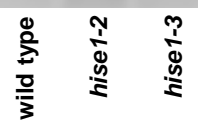

d

HMGR1

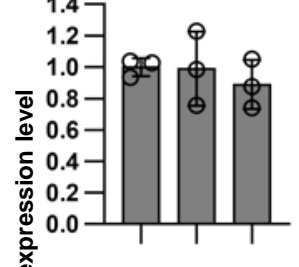

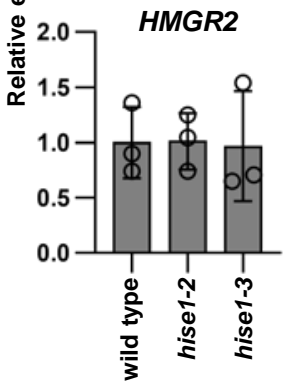

Figure 3 | Hyperaccumulation of HMGR proteins in hise1 mutant. a, A schematic view of biosynthesis of sterols via mevalonate pathway, isoprenoid pathway and sterol biosynthetic pathway. The enzymes indicated were detected by quantitative proteomics (see b and Supplementary Table 1). IPP, isopentenyl pyrophosphate; DMAPP, dimethylallyl pyrophosphate; FPP, farnesyl pyrophosphate. Lovastatin is a competitive inhibitor of HMGR and zaragozic acid is a competitive inhibitor of squalene synthase (SQS). b, Mass spectrometry for quantitative proteomics showing precursor ion intensities of enzymes that were detected in the microsomal fractions from wild type, hise1-2 and hise1-3 leaves. The precursor ion intensities were normalized by total peptide amounts. The intensity value of wild type was defined as 1.0. The averages of three biological replicates are shown. Error bars show standard deviations $(n=$ 3). Asterisks show significant differences between wild type and hise 1 mutants (two-sided t-test, $\mathrm{p}<0.01$ ). c, An immunoblot analysis of leaf extracts of wild type, hise1-2 and hise1-3 with anti-HMGR and anti-actin antibodies. Three biological replicates were performed with similar results. d, The expression levels of HMGR1 and HMGR2 mRNAs in wild type, hise1-2 and hise1-3 by quantitative real time PCR analysis. The averages of three biological replicates are shown. Error bars show standard deviations $(n=$ 3). The intensity value of wild type was defined as 1.0. Significant differences in the relative expression levels of HMGR1 and HMGR2 were examined ( $p>0.05$, Tukey's test). 

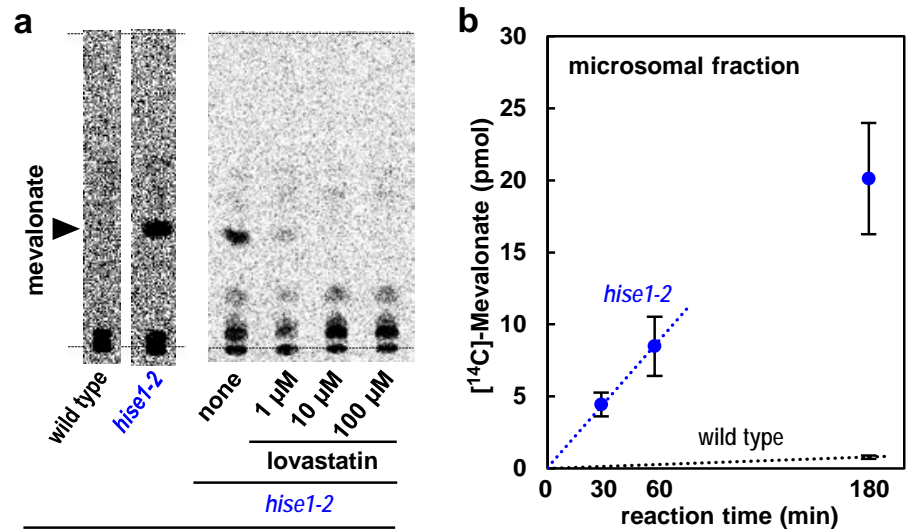

C

Specific activity

(pmol $\left[{ }^{14} \mathrm{C}\right]$-mevalonate $\mathrm{mg}$ protein ${ }^{-1} \mathrm{~min}^{-1}$ )

\begin{tabular}{ccc}
\hline & $\begin{array}{c}\text { crude } \\
\text { extract }\end{array}$ & $\begin{array}{c}\text { microsomal } \\
\text { fraction }\end{array}$ \\
\hline wild type & $0.79 \pm 0.11$ & n. d. \\
hise1-2 & $18.38 \pm 1.13$ & $26.49 \pm 4.90$
\end{tabular}

n. d., not detected

microsomal fraction

d

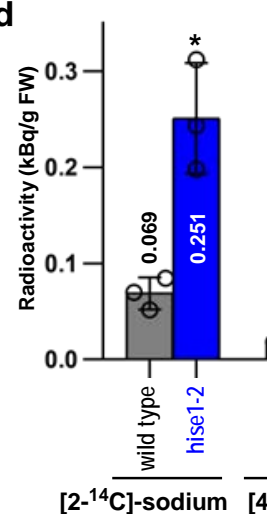

$\left[2-{ }^{14} \mathrm{C}\right]$-sodium

squalene

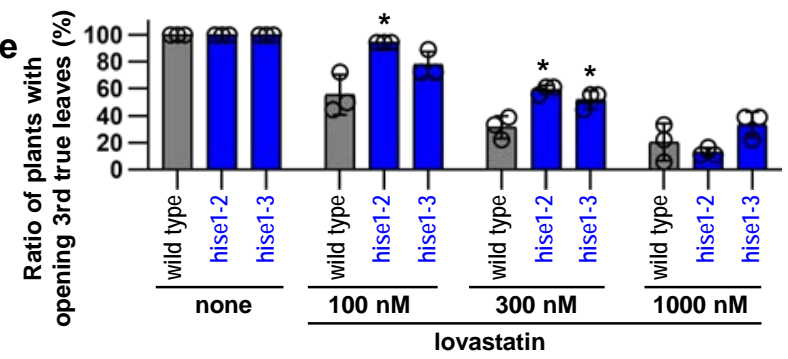

Figure 4 | hise1 mutant has dramatically higher sterol-producing activity than the wild type. a, Thin-layer chromatography showing $\left[{ }^{14} \mathrm{C}\right]$-mevalonate-producing activity from $\left[{ }^{14} \mathrm{C}\right]-\mathrm{HMG}-\mathrm{CoA}$ of microsomal fractions of the wild-type and hise 1 -2 leaves in the presence or absence of a HMGR inhibitor (lovastatin) for $60 \mathrm{~min}$ Two biological replicates were performed with similar results. b, Time-course data for $\left[{ }^{14} \mathrm{C}\right]$-mevalonate production with the wild-type and hise 1-2 microsomal fractions (5.6 $\mu \mathrm{g}$ protein). The averages of three biological replicates are shown. Error bars show standard deviations $(n=3)$. A significant difference between the wild type and hise1-2 was determined ( $p=3.6 \times 10^{-6}$, two-sided t-test). c, Specific HMGR activities of crude extracts and microsomal fractions from the wild-type and hise 1-2 leaves are shown. Three biological replicates were performed. Data represent average \pm standard deviation $(n=3)$. A significant difference between the wild type and hise 1 - 2 was determined ( $p=5.1 \times 10^{-10}$, two-sided t-test). d, In-vivo labeling with a radioactive $\left[{ }^{14} \mathrm{C}\right]$-sodium bicarbonate as an initial substrate for carbon fixation, $\left[2-{ }^{14} \mathrm{C}\right]$-sodium acetate as an acetyl-CoA precursor, and $\left[{ }^{14} \mathrm{C}\right]$-IPP as a squalene precursor. The incorporated radioactivity levels into squalene or sterol esters in the same fresh weights of wildtype and hise 1-2 leaves are shown. The averages of three biological replicates are shown. Error bars show standard deviations $(n=3)$. Asterisks exhibit a significant difference between wild type and hise1-2 ( $p<0.05$, two-sided t-test). e, Effects of lovastatin on the ratios of plants with opening third true leaves to 17 plants of wild type, hise1-2 and hise1-3. See Supplementary Fig. 6a for their plant growth. The averages of three biological replicates are shown. Error bars show standard deviations $(n=3)$. Asterisks exhibit a significant difference between wild type and hise 1 mutants ( $p<0.05$, two-sided t-test). 

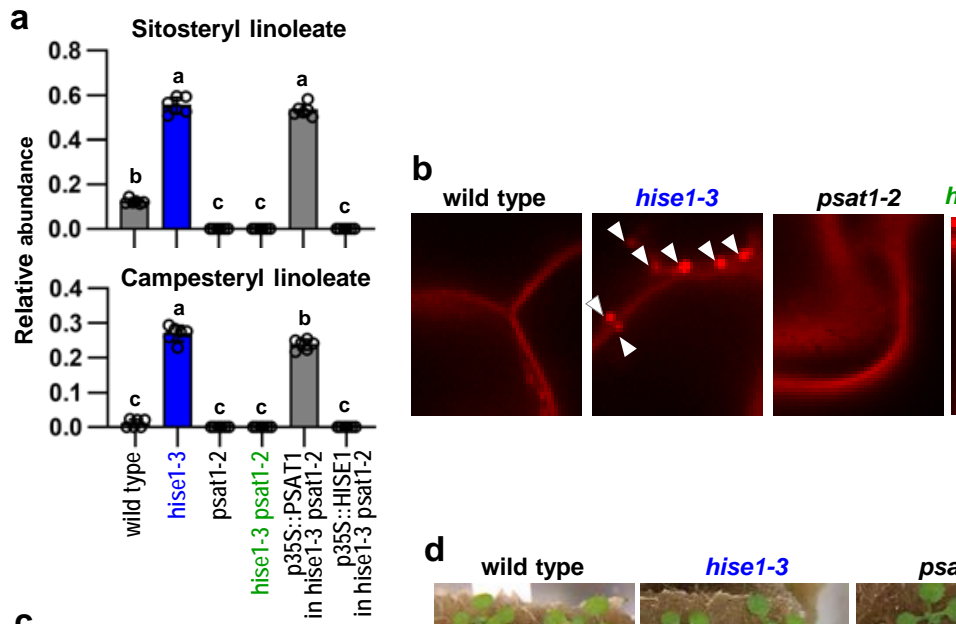

p35S::PSAT1

p35S::HiSE1
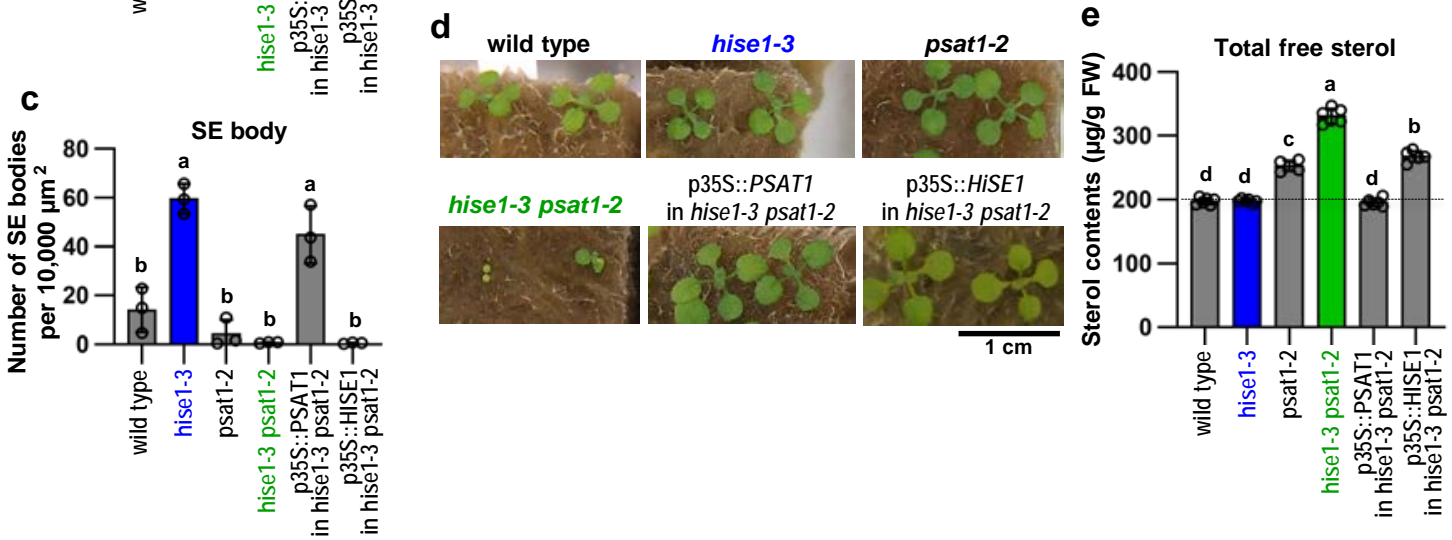

f

\begin{tabular}{crrrrrr}
\hline & wild type & hise1-3 & psat1-2 & hise1-3psat1-2 & $\begin{array}{c}\text { 35S::PSAT1 } \\
\text { in } \\
\text { hise1-3psat1-2 }\end{array}$ & $\begin{array}{c}\text { 35S::HISE1 } \\
\text { in } \\
\text { hise1-3psat1-2 }\end{array}$ \\
\hline beta-sitosterol & $145.33 \pm 3.54$ & $143.99 \pm 2.91$ & $188.49 \pm 6.21$ & $220.08 \pm 6.04$ & $141.31 \pm 4.46$ & $198.24 \pm 6.41$ \\
campesterol & $41.56 \pm 1.67$ & $42.16 \pm 1.24$ & $52.65 \pm 2.09$ & $88.07 \pm 5.75$ & $42.93 \pm 1.55$ & $56.46 \pm 1.74$ \\
stigmastanol & $1.01 \pm 0.07$ & $0.45 \pm 0.02$ & $1.25 \pm 0.06$ & $1.23 \pm 0.14$ & $0.51 \pm 0.04$ & $1.30 \pm 0.05$ \\
cholesterol & $5.76 \pm 0.60$ & $5.64 \pm 0.32$ & $7.13 \pm 0.39$ & $8.60 \pm 0.79$ & $5.62 \pm 0.26$ & $7.21 \pm 0.30$ \\
brassicasterol & $2.39 \pm 0.09$ & $2.65 \pm 0.10$ & $3.30 \pm 0.19$ & $5.15 \pm 0.49$ & $2.59 \pm 0.14$ & $3.47 \pm 0.20$ \\
stigmasterol & $0.71 \pm 0.07$ & $0.58 \pm 0.19$ & $0.86 \pm 0.10$ & $3.21 \pm 1.15$ & $0.61 \pm 0.05$ & $0.96 \pm 0.11$ \\
cycloartenol & $0.67 \pm 0.02$ & $2.21 \pm 0.15$ & $0.89 \pm 0.08$ & $2.97 \pm 0.41$ & $1.81 \pm 0.10$ & $1.17 \pm 0.09$ \\
24-methylenecycloartanol & $0.17 \pm 0.01$ & $0.49 \pm 0.02$ & $0.31 \pm 0.03$ & $1.68 \pm 0.18$ & $0.39 \pm 0.01$ & $0.42 \pm 0.02$ \\
\hline
\end{tabular}

Values are given in $\mu \mathrm{g} / \mathrm{g} \mathrm{FW}$.

Figure 5 | Deficiency of PSAT1 causes defects in SE-body formation and plant growth of hise1. The wild type, two single mutants (hise1-3 and psat1-2), the double mutant (hise1-3 psat1-2), and the double mutant rescued by expressing either p35S::HiSE1 or p35S::PSAT1 were used. a, The contents of sitosteryl linoleate and campesteryl linoleate in 2-week-old leaves are shown as relative values of signal intensities of $[\mathrm{M}+\mathrm{NH} 4]^{+}$species of sterol esters to that of $[\mathrm{M}+\mathrm{H}]^{+}$of internal standard (1,2-didecanoyl-sn-glycero-3phosphocholine). The averages of six biological replicates are shown. Error bars show standard deviations $(n=6)$. Different letters exhibit significant differences ( $p<0.05$, Tukey's test). $\mathbf{b}$, Confocal laser-scanning microscopic images of leaf cells stained with Nile red. Arrowheads show SE bodies. Three biological replicates were performed with similar results. c, A quantitative analysis of SE-body densities in 2-week-old leaves. The averages of six biological replicates are shown. Error bars show standard deviations $(n=6)$. Different letters exhibit significant differences ( $p<0.05$, Tukey's test). d, Growth phenotypes of 2-week-old seedlings of each line on rock wools. Three biological replicates were performed with similar results. e, Total contents of free sterols in 2-week-old juvenile leaves. Values are given in $\mu \mathrm{g} / \mathrm{g}$ fresh weight (FW). A dotted line shows the total free sterol level of the wild type. The averages of biological replicates with error bars (standard deviations). Wild type $(n=6)$, hise1-3 $(n=6)$, psat1-2 $(n=5)$, hise1-3 psat1-2 $(n=6)$ and p35S::PSAT1 in hise1-3 psat1-2 $(n=6)$, and p35S::HiSE1 in hise1-3 psat1-2 $(n=5)$. Different letters exhibit significant differences $(p<0.05$, Tukey-Kramer method). f, Free sterol contents of each line. Sterol moiety was annotated based on the $\mathrm{m} / \mathrm{z}$ value of fragment ion detected in MS/MS spectra and literature data on phytosterols. Data represent average \pm standard deviation. Biological replicates were performed; wild type $(n=6)$, hise1-3 $(n=6)$, psat1-2 $(n=5)$, hise1-3 psat1-2 $(n=6)$ and p35S::PSAT1 in hise1-3 psat1-2 $(n=6)$, and p35S::HiSE1 in hise1-3 psat1-2 $(n=5)$. 
a

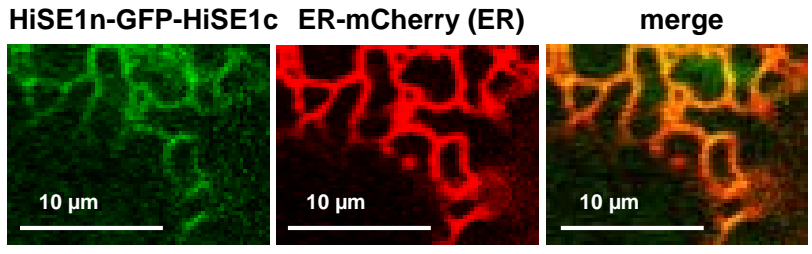

b PSAT1-TagRFP HiSE1n-GFP-HiSE1c

merge
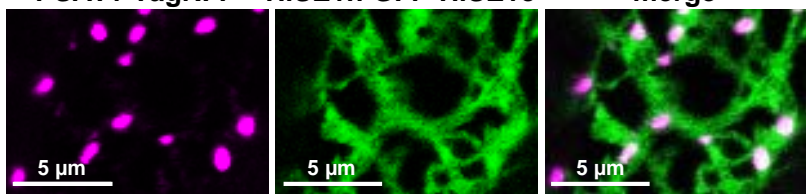

PSAT1-TagRFP

GFP-HDEL (ER)
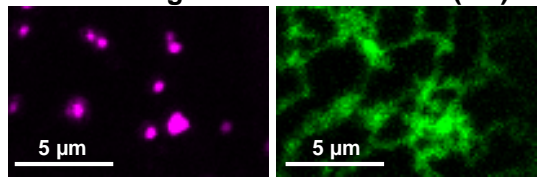

merge

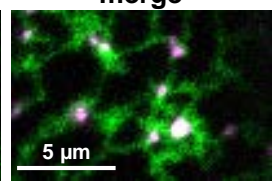

PSAT1-TagRFP

GFP-RHD3 (ER)
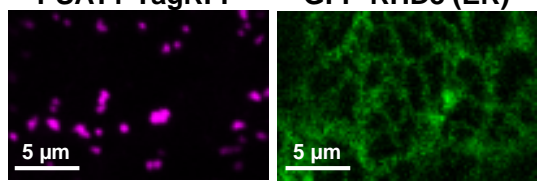

merge

C

Labeling rates of PSAT1 punctate structures

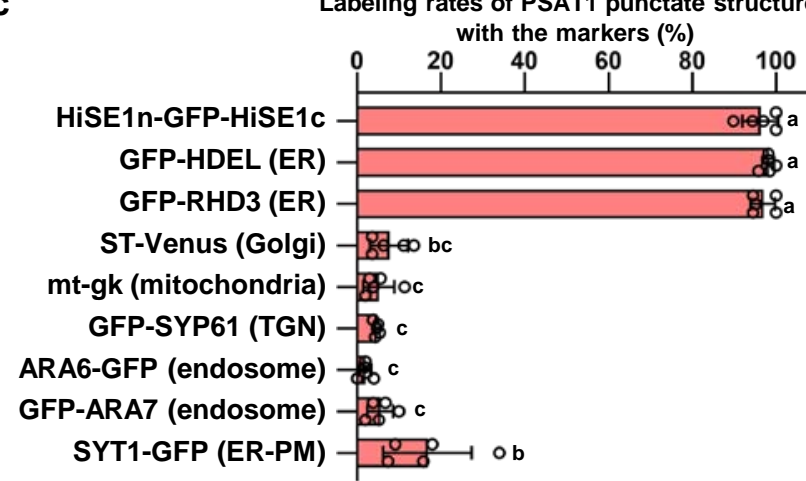

Figure 6 | HiSE1 localizes to the ER, while PSAT1 localizes to the ER microdomains. a, Transient expression of HiSE1n-GFP-HiSE1c with the ER marker ER-mCherry in $N$. benthamiana leaves. b, Transient expression of PSAT1TagRFP with each of HiSE1n-GFP-HiSE1c, the ER marker GFP-HDEL, and the ER-membrane marker GFP-RHD3 in $N$. benthamiana leaves. c, Labeling rates of PSAT1 punctate structures with the markers indicated. PM, Plasma membrane. The averages with error bars (standard deviations) of five biological replicates are shown. In each experiment, 50 to 100 PSAT1 punctate structures were analyzed. Different letters exhibit significant differences ( $p<0.05$, Tukey's test). 


\section{HiSE1-depedent fail-safe regulatory system for sterol homeostasis}

Process 1

Down-regulation of HMGR

to prevent overproduction of sterols

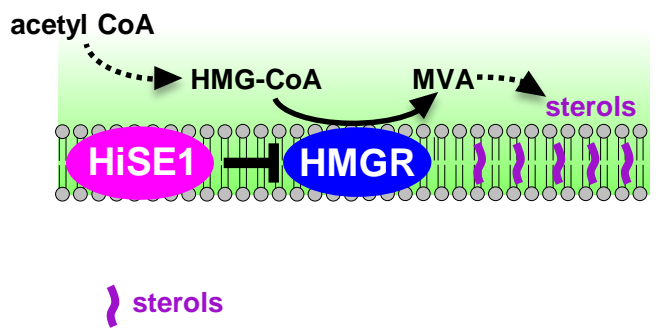

sterols
Process 2

Detoxification of excess sterols and their segregation into SE bodies
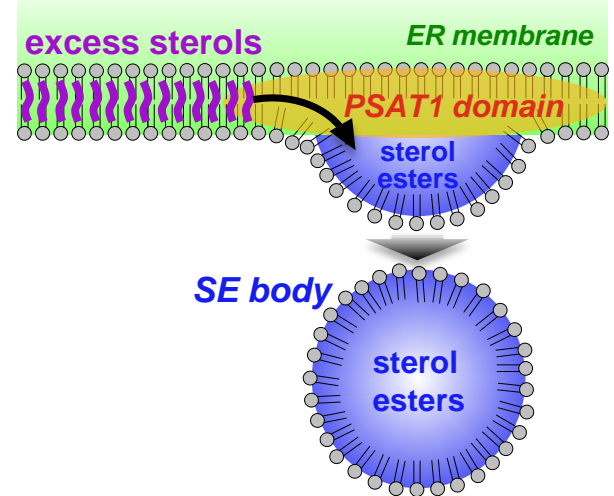

Figure 7 | A hypothetical model of a HiSE1-depedent fail-safe regulatory system for sterol homeostasis via HMGR down-regulation. The regulatory system is composed of two processes: 1) HiSE1-mediated downregulation of HMGR to prevent sterol overproduction on the ER and 2) Detoxification of excess amounts of sterols on the PSAT1-localized ER microdomains and segregation of sterol esters into SE bodies. MVA, mevalonate. 


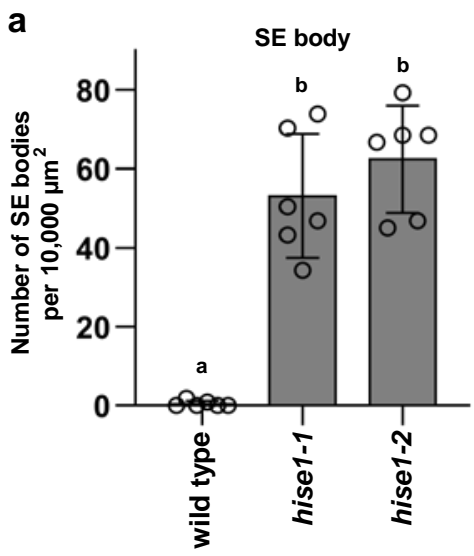

b
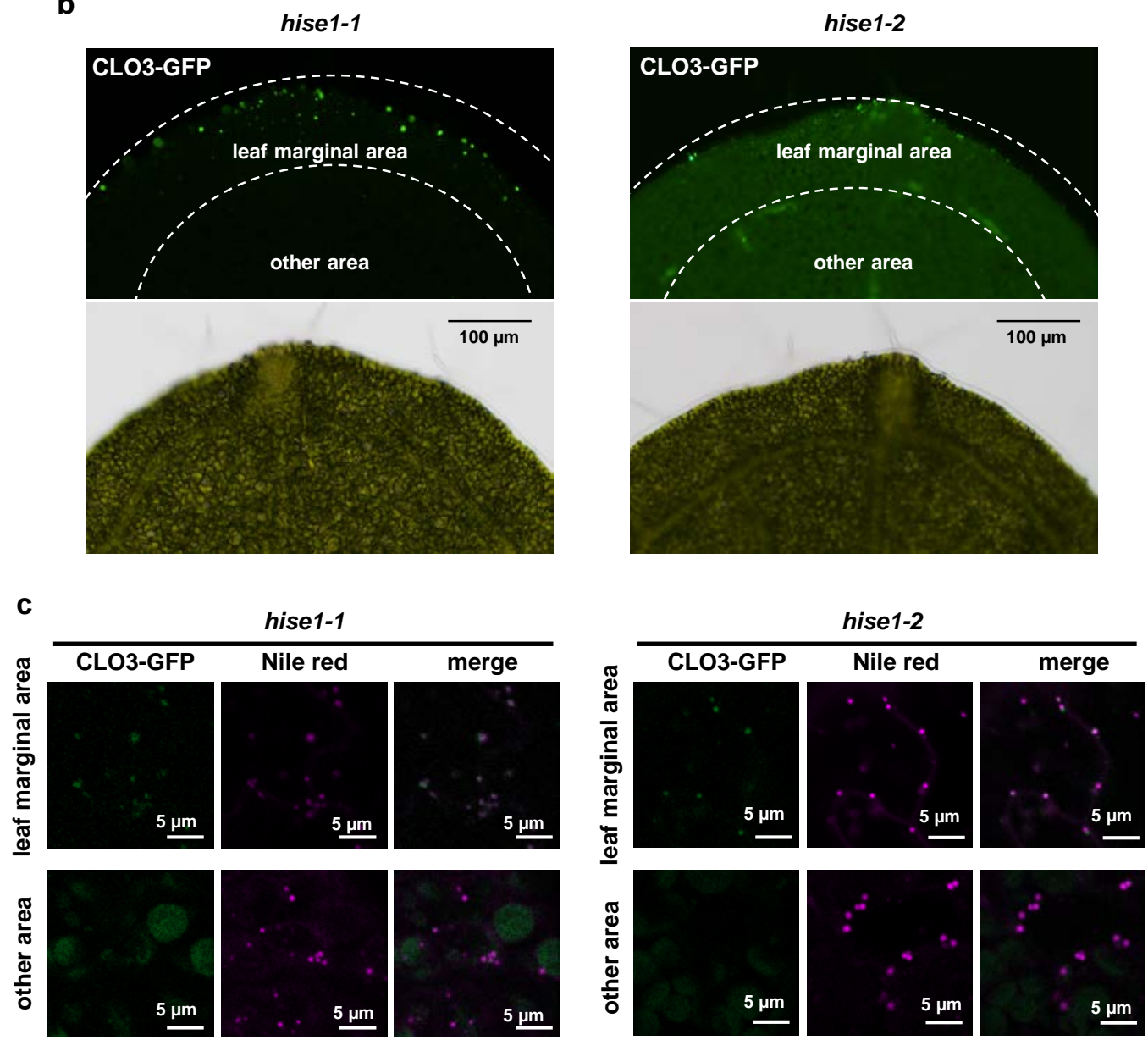

Supplementary Figure 1 | CLO3-GFP-positive and CLO3-GFP-negative populations of SE bodies in hise1-1 and hise1-2 leaves. a, Quantitative analysis of SE body densities in fluorescence microscopic images of juvenile leaves of 2-week-old wild-type, hise1-1, and hise1-2 plants containing the CLO3-GFP transgene. The averages of six biological replicates are shown. Error bars represent standard deviation $(n=6)$. Different letters indicate significant differences ( $p<0.05$; Tukey's test). $\mathbf{b}$, GFP fluorescence and bright field images of the first true leaves of 2-week-old hise1-1 and hise1-2 plants containing the CLO3-GFP transgene. Three biological replicates were performed with similar results. c, Confocal laser-scanning microscopic images showing CLO3-GFP and Nile red-labeled SE bodies of the first true leaves in b. Note that SE bodies are separated into two populations; a CLO3-GFP-positive population in the leaf marginal areas and a CLO3-GFP-negative population throughout the leaves. Three biological replicates were performed with similar results. 
a
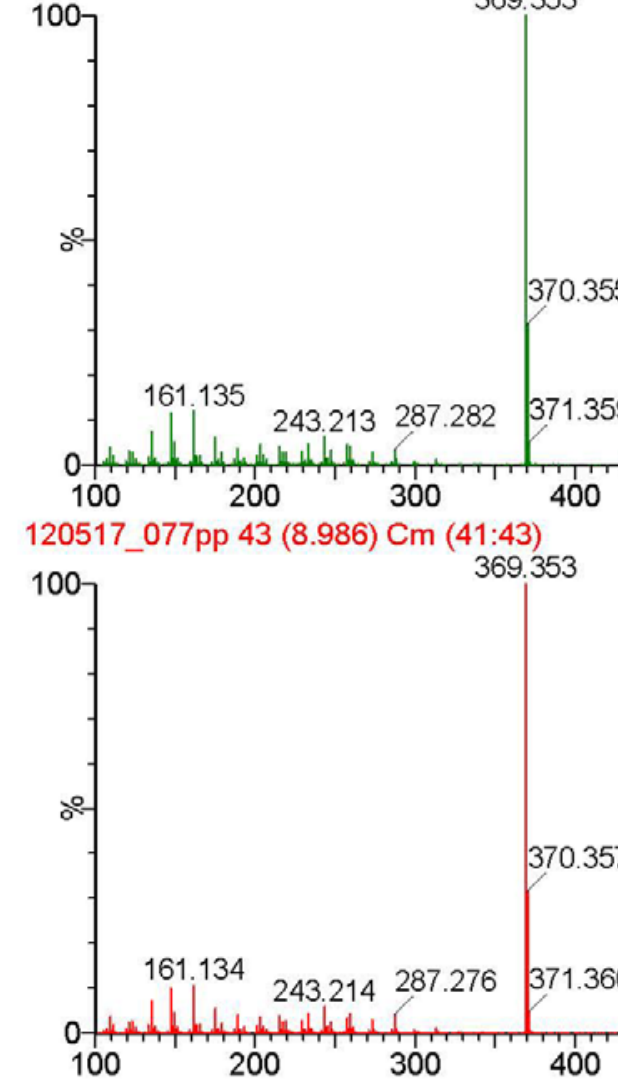

\section{Cholesteryl linoleate}

(standard, $\left[\mathrm{M}+\mathrm{NH}_{4}\right]^{+}$)

$\mathrm{m} / \mathrm{z} 666.62$

Cholesteryl palmitate

(standard, $\left[\mathrm{M}+\mathrm{NH}_{4}\right]^{+}$)

$\mathrm{m} / \mathrm{z} 642.62$

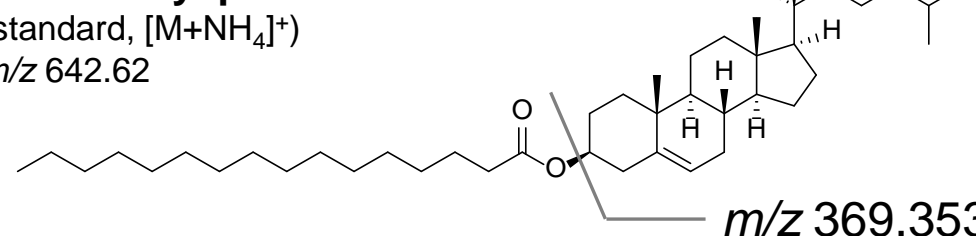

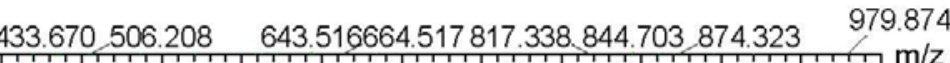
500600 700 800

3.73 e 5

\section{$m / z 369.353$}

\section{C}

\section{7_069pp $333(9.022) \mathrm{Cm}(332: 334)$}

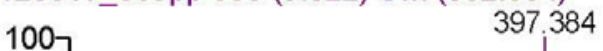

\section{Sitosteryl linolenate}

(a major hise1-specific lipid, [M+NH$\left.]_{4}\right]^{+}$) $\mathrm{m} / \mathrm{z} 692.63$

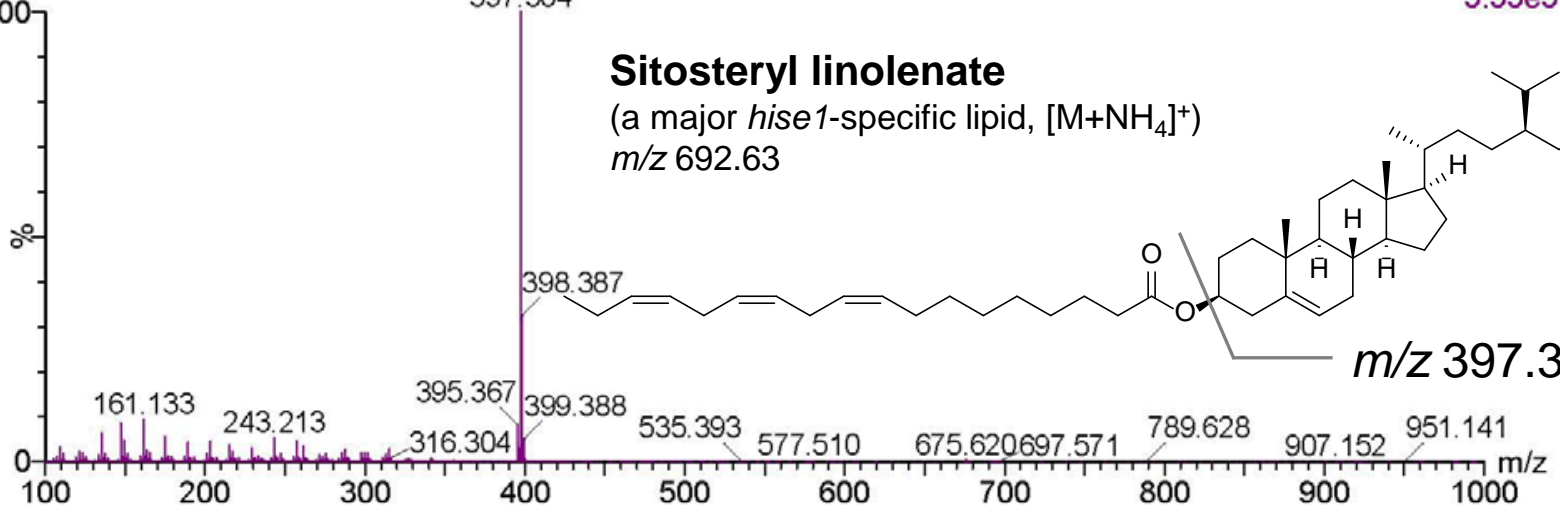

Supplementary Figure 2 | Structural characterization of sterol esters via tandem mass spectrometry (MS/MS) in the positive ion mode. MS/MS spectra of two standard sterol esters and a major hise1-specific sterol ester are shown. The $\mathrm{m} / \mathrm{z}$ value of the precursor ion, $\left[\mathrm{M}+\mathrm{NH}_{4}\right]^{+}$, was subjected to MS/MS analysis. A fragment ion attributable to sterol moiety formed by the neutral loss of fatty acid moiety was observed as a base peak in the MS/MS spectrum. Three biological replicates were performed with similar results. 

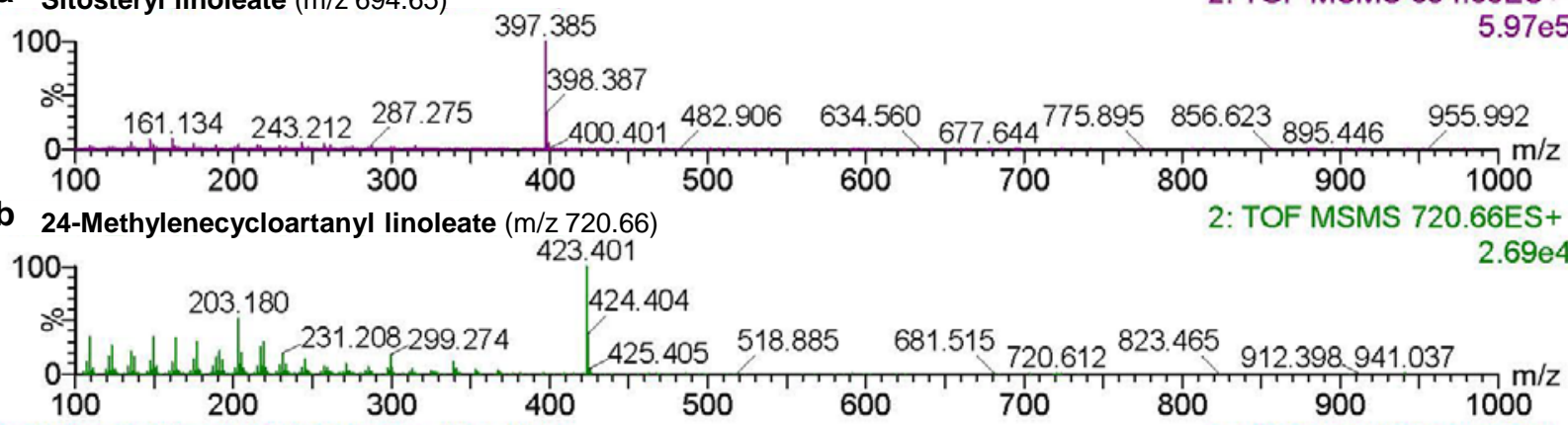

C Campesteryl linoleate $(\mathrm{m} / \mathrm{z} 680.63)$

2: TOF MSMS 680.63ES+
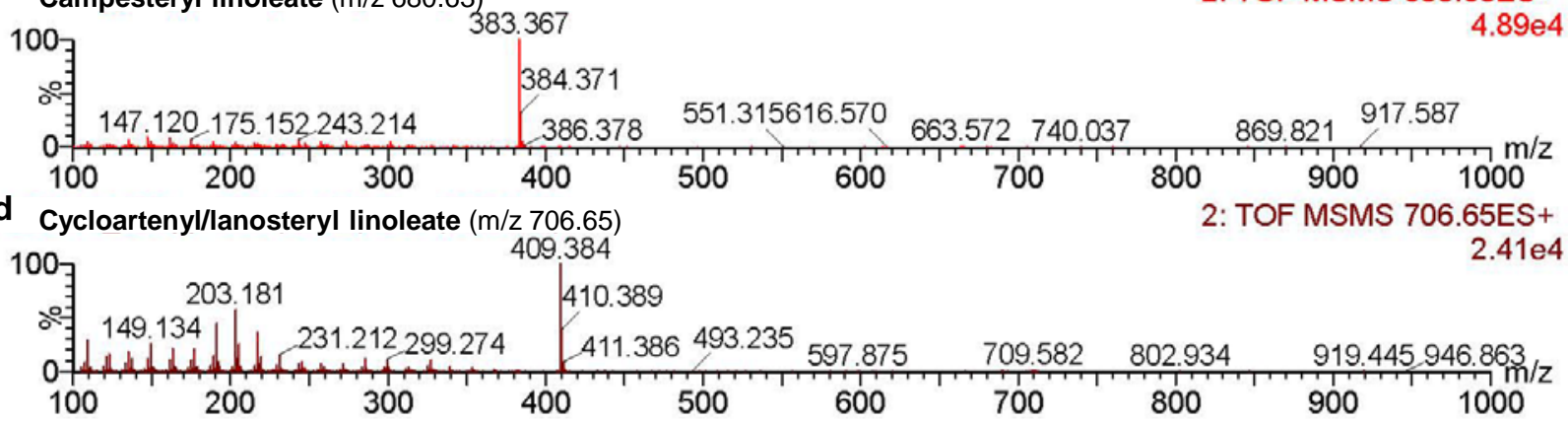

e Sitosteryl linolenate $(\mathrm{m} / \mathrm{z}$ 692.63)

2: TOF MSMS 692.63ES+

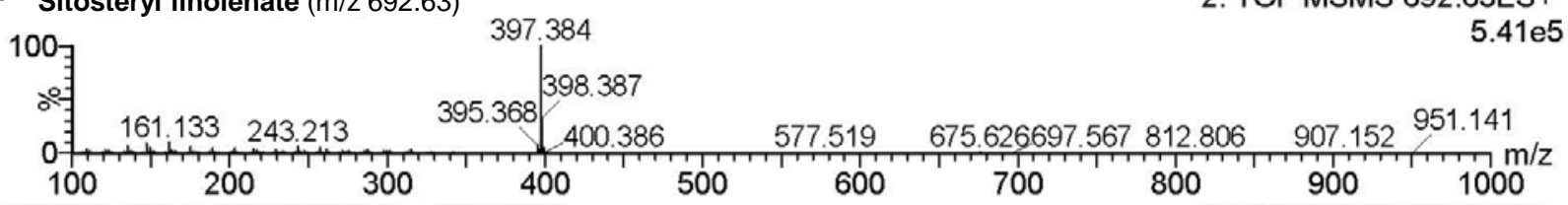

f 24-Methylenecycloartanyl linolenate $(\mathrm{m} / \mathrm{z} 718.65)$

2: TOF MSMS 718.65ES+

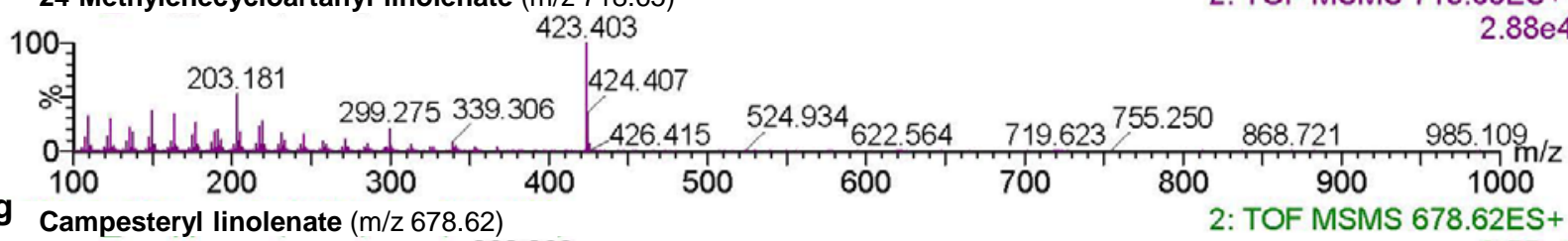

g Campesteryl linolenate $(\mathrm{m} / \mathrm{z} 678.62)$

2: TOF MSMS 678.62ES+

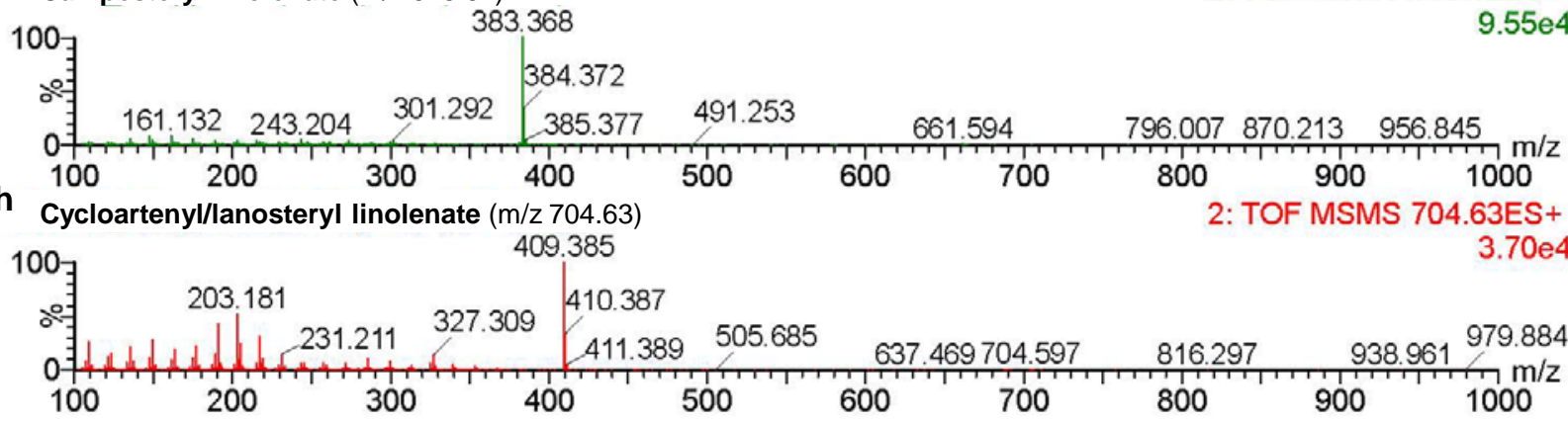

Supplementary Figure 3 | MS/MS spectra of a series of sterol esters accumulated abundantly in hise1 plants. MS/MS spectra of hise1-specific sterol esters are shown. The $m / z$ value of the precursor ion, $\left[\mathrm{M}+\mathrm{NH}_{4}\right]^{+}$, was subjected to $\mathrm{MS} / \mathrm{MS}$ analysis. A fragment ion attributable to sterol moiety formed by the neutral loss of fatty acid moiety was observed as a base peak in the MS/MS spectrum. Three biological replicates were performed with similar results. 


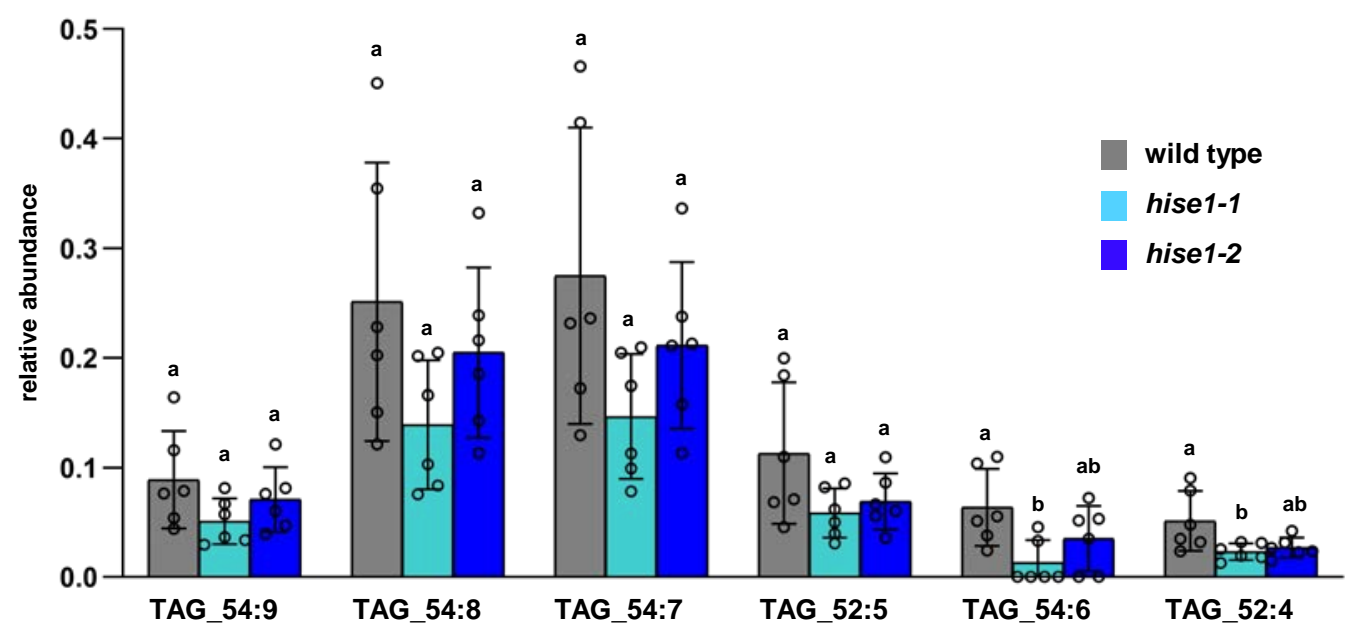

Supplementary Figure 4 | Liquid chromatography-mass spectrometry (LC-MS) analysis of triacylglycerols (TAGs) isolated from the juvenile leaves of two hise1 mutants and the wild type. Contents of TAGs are expressed as signal intensities of $[\mathrm{M}+\mathrm{NH} 4]^{+}$species relative to that of $[\mathrm{M}+\mathrm{H}]^{+}$of the internal standard (1,2-didecanoyl-sn-glycero-3phosphocholine). For example, TAG_52:5 has 52 carbons and 5 double bonds. The averages of six biological replicates are shown. Error bars represent standard deviation $(n=6)$. Different letters indicate significant differences $(p<0.05$; Tukey's test). 
$\mathbf{a}$

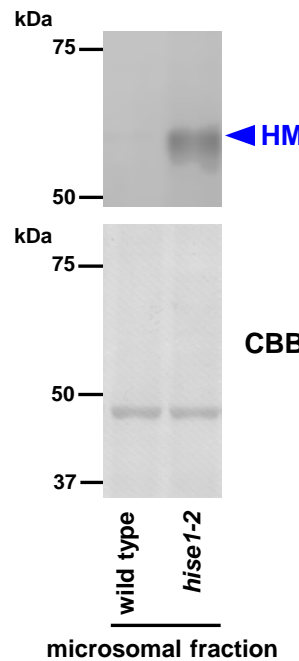

b

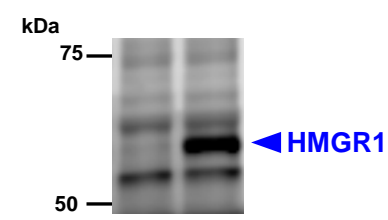

$\mathrm{kDa}$

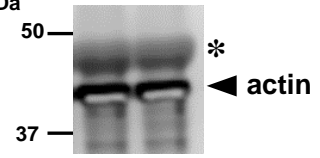

Supplementary Figure 5 | Microsomal fractions and crude extracts from the wild type and hise1-2 used for measuring HMGR activities. a, An immunoblot of microsomal fractions of the wild type and hise1-2 with anti-HMGR antibody. The blotting membrane was stained with Coomassie Brilliant Blue (CBB). Three biological replicates were performed with similar results. $\mathbf{b}$, An immunoblot of crude leaf extracts of the wild type and hise1-2 with either anti-HMGR antibody or anti-actin antibody. An asterisk shows a non-specific band. Three biological replicates were performed with similar results. 

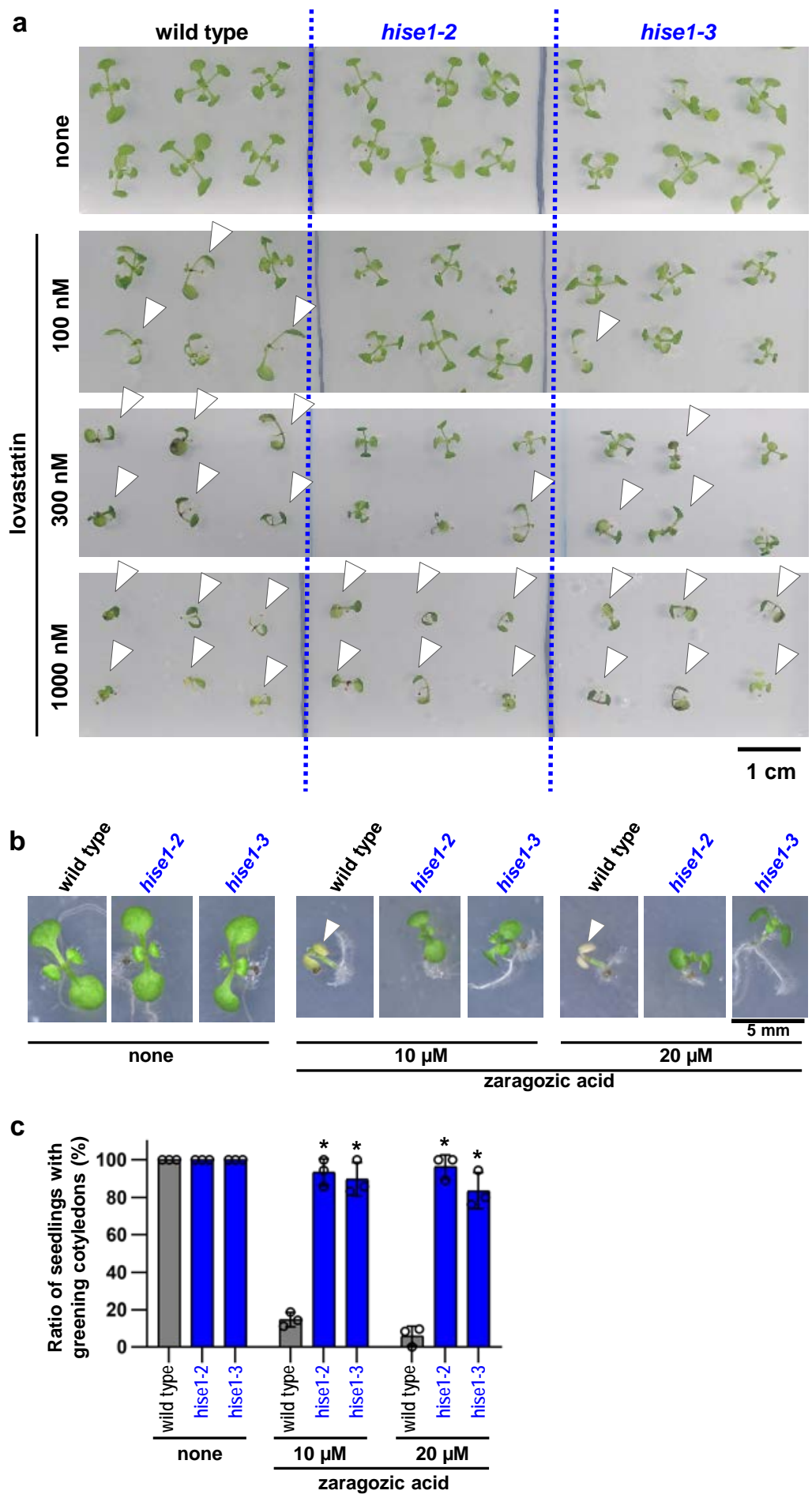

Supplementary Figure 6 | Effects of the HMGR inhibitor lovastatin and the SQS inhibitor zaragozic acid on growth of the wildtype and hise1 plants. a, Two-week-old plants of the wild type and hise1 mutant alleles (hise1-2 and hise1-3) on the agar plates in the absence and presence of the HMGR inhibitor lovastatin. White arrowheads show plants that were unable to open the third true leaves. Three biological replicates were performed with similar results. b. Ten-day-old seedlings of the wild type and hise1 mutant alleles on the agar plates in the absence and presence of the SQS inhibitor zaragozic acid. White arrowheads show senesced cotyledons. Three biological replicates were performed with similar results. c, Effects of zaragozic acid on the ratio of seedlings with greening cotyledons to 15 seedlings of the wild type, hise1-2 and hise1-3. See Supplementary Fig. 6b for their plant growth. The averages of three biological replicates are shown. Error bars show standard deviations $(n=3)$. Asterisks show significant differences between wild type and hise 1 mutants ( $p<0.05$, two-sided t-test). 


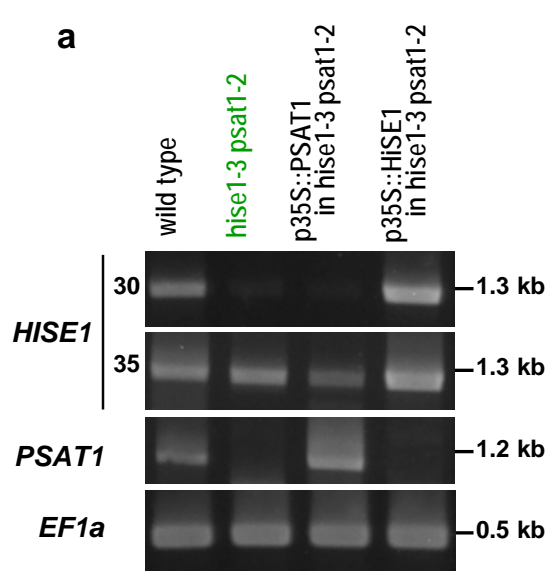

b

\begin{tabular}{|c|c|c|c|c|c|c|}
\hline & wild type & hise1-3 & psat1-2 & $\begin{array}{l}\text { hise1-3 } \\
\text { psat1-2 }\end{array}$ & $\begin{array}{c}\text { 35S::PSAT1 } \\
\text { in } \\
\text { hsie1-3 } \\
\text { psat1-2 }\end{array}$ & $\begin{array}{c}\text { 35S::HISE1 } \\
\text { in } \\
\text { hise1-3 } \\
\text { psat1-2 }\end{array}$ \\
\hline sitosteryl linoleate & $0.12 \pm 0.01$ & $0.55 \pm 0.04$ & n. d. & $0 \pm 0$ & $0.53 \pm 0.03$ & n. d. \\
\hline sitosteryl linolenate & $0.15 \pm 0.01$ & $0.83 \pm 0.05$ & n. d. & $0 \pm 0$ & $0.70 \pm 0.03$ & n. d. \\
\hline campesteryl linoleate & $0.01 \pm 0.01$ & $0.27 \pm 0.02$ & n. d. & n. d. & $0.24 \pm 0.01$ & n. d. \\
\hline campesteryl linolenate & $0.02 \pm 0.01$ & $0.27 \pm 0.01$ & n. d. & n. d. & $0.22 \pm 0.02$ & n. d. \\
\hline stigmasteryl linolenate & n. d. & $0.28 \pm 0.02$ & n. d. & n. d. & $0.21 \pm 0.01$ & n. d. \\
\hline cycloartenyl/ lanosteryl linoleate & n. d. & $0.14 \pm 0.01$ & n. d. & n. d. & $0.15 \pm 0.01$ & n. d. \\
\hline cycloartenyl/ lanosteryl linolenate & n. d. & $0.19 \pm 0.02$ & $0 \pm 0$ & n. d. & $0.20 \pm 0.01$ & $0 \pm 0$ \\
\hline 24-methylenecycloartanyl linoleate & n. d. & $0.07 \pm 0.01$ & n. d. & n. d. & $0.07 \pm 0.00$ & n. d. \\
\hline 24-methylenecycloartanyl linolenate & n. d. & $0.09 \pm 0.01$ & n. d. & n. d. & $0.08 \pm 0.01$ & n. d. \\
\hline
\end{tabular}

Supplementary Figure 7 | Sterol ester contents in leaves of $A$. thaliana mutant lines and the wild type. a, RT-PCR of HiSE1, PSAT1, and EF1a (control) transcripts in 2-week-old seedlings of each line indicated. Numbers of PCR cycles for each gene are shown. Three biological replicates were performed with similar results. b, Sterol ester contents in juvenile leaves of each line. Signal intensities of $[\mathrm{M}+\mathrm{NH} 4]^{+}$species of sterol esters relative to that of $[\mathrm{M}+\mathrm{H}]^{+}$of the internal standard $(1,2-$ didecanoyl-sn-glycero-3-phosphocholine) are shown.

Data represent average \pm standard deviation. Six biological replicates were performed. n.d., not detected. 
a

HiSE1n-GFP-HiSE1c

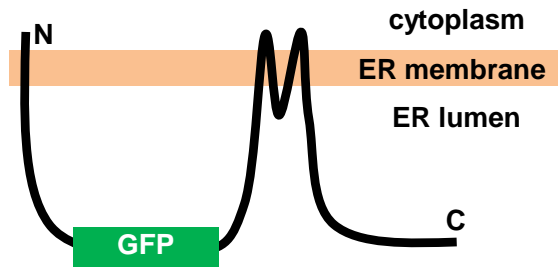

b

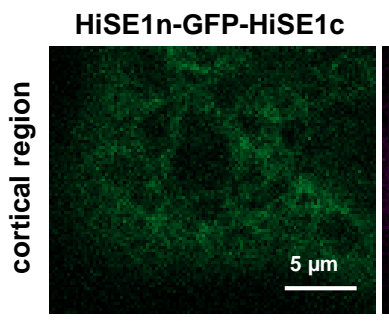

FM4-64 (PM)
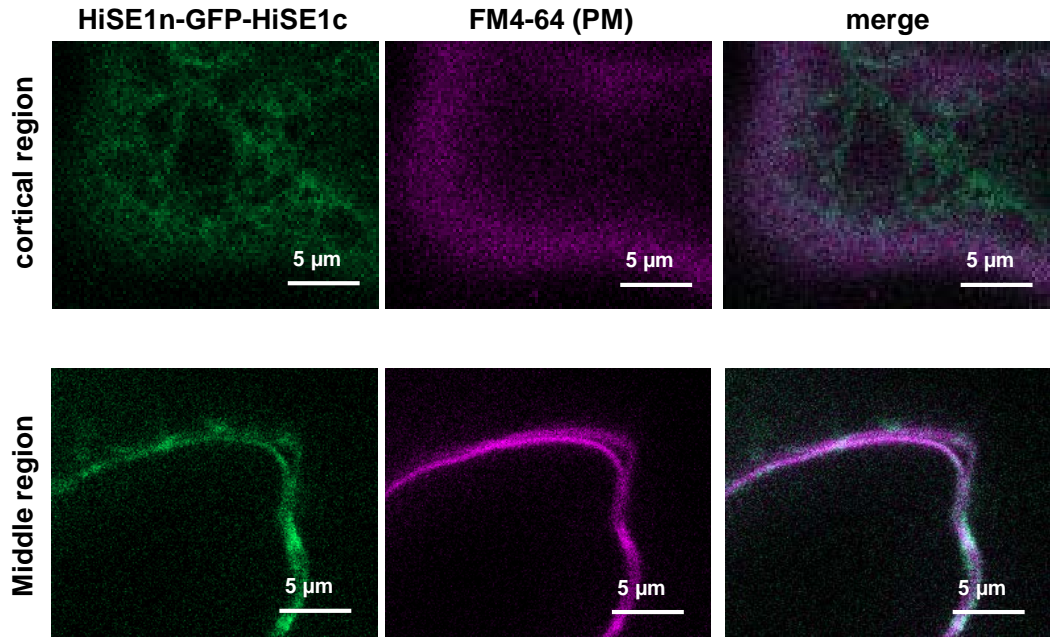

Supplementary Figure 8 | No overlap of HiSE1n-GFP-HiSE1c signals with the FM4-64-labeled plasma membrane. a, A schematic view of the GFP fusion, HiSE1n-GFP-HiSE1c. The GFP fragment was inserted between the 183th and 184th amino acid of HiSE1. b, N. benthamiana leaves expressing HiSE1n-GFP-HiSE1c were stained with FM4-64 to visualize the plasma membrane (PM). Fluorescence images of the cortical region (upper) and middle region (lower) of the cell are shown. Three biological replicates were performed with similar results. 

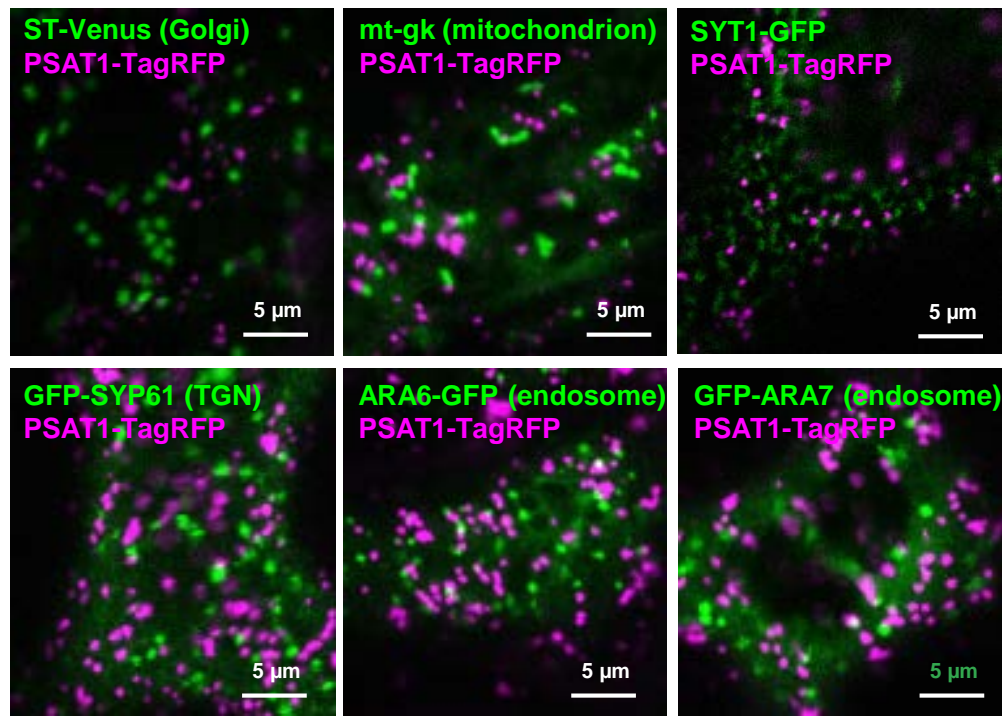

Supplementary Figure 9 | Localization of PSAT1 to the endoplasmic reticulum (ER) microdomains. The PSAT1-TagRFP fusion was transiently co-expressed with each organelle marker in $N$. benthamiana leaves. Different organelle markers used included the Golgi marker ST-Venus, the mitochondrial marker mt-gk, the ER-plasma membrane-contact site marker SYT1-GFP, the trans-Golgi-network (TGN) marker GFP-SYP61, and two endosome markers ARA6-GFP and GFP-ARA7. Three biological replicates were performed with similar results. 


\section{Supplementary Table 1 | Protein levels of sterol biosynthetic enzymes in microsomal fractions of leaves of wild type, hise1-2 and hise1-3.}

\begin{tabular}{|c|c|c|c|c|c|}
\hline \multirow[b]{2}{*}{ Protein } & \multirow[b]{2}{*}{ Accession } & \multirow[b]{2}{*}{ Description } & \multicolumn{3}{|c|}{ Precursor ion intensity } \\
\hline & & & wild type & hise1-2 & hise1-3 \\
\hline ACAT2 & AT5G48230.2 & acetoacetyl-CoA thiolase 2 & $1 \pm 0.12$ & $1.02 \pm 0.24$ & $1.20 \pm 0.19$ \\
\hline HMGS & AT4G11820.1 & hydroxymethylglutaryl-CoA synthase & $1 \pm 0.10$ & $1.06 \pm 0.16$ & $1.03 \pm 0.07$ \\
\hline HMGR1 & AT1G76490.1 & 3-hydroxy-3-methylglutaryl CoA reductase 1 & $1 \pm 0.77$ & $129.06 \pm 2.91 *$ & $70.80 \pm 12.85^{*}$ \\
\hline HMGR2 & AT2G17370.1 & 3-hydroxy-3-methylglutaryl-CoA reductase 2 & $1 \pm 2.45$ & $224.64 \pm 42.62 *$ & $137.33 \pm 53.19 *$ \\
\hline MVD1 & AT2G38700.1 & mevalonate diphosphate decarboxylase 1 & $1 \pm 0.29$ & $1.61 \pm 0.22 *$ & $1.84 \pm 0.26^{*}$ \\
\hline FPS1 & AT5G47770.1 & farnesyl diphosphate synthase 1 & $1 \pm 0.15$ & $1.19 \pm 0.14$ & $1.39 \pm 0.39$ \\
\hline FPS2 & AT4G17190.1 & farnesyl diphosphate synthase 2 & $1 \pm 1.74$ & $2.23 \pm 1.76$ & $1.70 \pm 1.88$ \\
\hline SQS1 & AT4G34640.1 & squalene synthase 1 & $1 \pm 0.07$ & $1.03 \pm 0.05$ & $1.02 \pm 0.11$ \\
\hline SQE1 & AT1G58440.1 & squalene epoxidase 1 & $1 \pm 2.45$ & $2.03 \pm 4.97$ & 0 \\
\hline SQE3 & AT4G37760.1 & squalene epoxidase 3 & $1 \pm 1.10$ & $1.74 \pm 0.16$ & $1.52 \pm 1.68$ \\
\hline LAS1 & AT3G45130.1 & lanosterol synthase 1 & $1 \pm 0.35$ & $0.55 \pm 0.15$ & $0.83 \pm 0.23$ \\
\hline CAS1 & AT2G07050.1 & cycloartenol synthase 1 & $1 \pm 0.08$ & $0.92 \pm 0.10$ & $1.03 \pm 0.04$ \\
\hline SMT1 & AT5G13710.2 & sterol methyltransferase 1 & $1 \pm 0.12$ & $0.98 \pm 0.04$ & $1.31 \pm 0.16$ \\
\hline CYP51G1 & AT1G11680.1 & cytochrome P450 51G1 & $1 \pm 0.11$ & $1.04 \pm 0.07$ & $1.31 \pm 0.21$ \\
\hline HYD1 & AT1G20050.1 & C-8,7 sterol isomerase & $1 \pm 0.12$ & $1.22 \pm 0.04 *$ & $1.30 \pm 0.07^{*}$ \\
\hline SMT2 & AT1G20330.1 & sterol methyltransferase 2 & $1 \pm 0.03$ & $1.19 \pm 0.09 *$ & $1.33 \pm 0.11 *$ \\
\hline SMT3 & AT1G76090.1 & sterol methyltransferase 3 & $1 \pm 0.11$ & $1.19 \pm 0.10$ & $0.91 \pm 0.39$ \\
\hline DWF5 & AT1G50430.1 & delta5,7-sterol delta7 reductase & $1 \pm 0.12$ & $0.86 \pm 0.03$ & $0.86 \pm 0.07$ \\
\hline DWF1 & АT3G19820.3 & delta24-sterol reductase & $1 \pm 0.06$ & $0.92 \pm 0.06$ & $1.13 \pm 0.14$ \\
\hline
\end{tabular}

The intensity value of wild type is defined as 1 . Data represent average \pm standard deviation. Three biological replicates were performed. Asterisks show significant differences between wild type and hise 1 mutants (two-sided t-test, $p<0.01$ ). See Methods for details. 